\title{
A Fluidic Alternative to the EM Drive Propulsion Concept: The Orthogonal Fluid Flow Propulsion Concept
}

\author{
Edward A. Walker \\ Correspondence: Edward A. Walker, Florida Memorial University; 15800 N.W. 42 ${ }^{\text {nd }}$ Ave. Miami Garden FL. 33054, \\ Mathematics Department, USA.
}

Received: September 25, 2017

Accepted: January 14, 2018 Online Published: January 24, 2018

doi:10.11114/set.v5i1.2669

URL: https://doi.org/10.11114/set.v5i1.2669

\begin{abstract}
A theoretical space propulsion concept in which a system does not lose its ionized propellant is introduced. Due to the fact that no propellant mass is lost by the system, the theoretical propulsion concept is synonymous to a form of propellant less propulsion similarly to the EM Drive. Therefore, a summarization of the EM drive including a brief description of other advances in ion propulsion is given along with a comparison to the new theoretical propulsion concept to be introduced. The orthogonal fluid flow propulsion concept or OFFPC describes orthogonal fluid flows of ionized propellant injected by orthogonal nozzles into an enclosed chamber where the ionized propellant is governed by electric forces generated by the chamber walls and will be mathematically shown to generate thrust. Inevitably, Newton's third law and momentum conservation are addressed in relation to the OFFPC. Specifically, aerodynamic momentum conservation in a controlled volume system is used to show that the OFFPC obeys momentum conservation after elucidating the role of Newton's third law within the theoretical system. The capability of a propulsion system incorporating the OFFPC to regenerate a portion of its electric energy (and voltage) similarly to an automobile alternator will be mathematically expressed. Thus, a description of how the system exhausts energy as with any conventional system is given to show that a propulsion system incorporating the OFFPC is not a perpetual motion device. Lastly, a mathematical demonstration of a hypothetical vehicle is given to show quantitative results and theoretical performance figures produced by the OFFPC.
\end{abstract}

Keywords: EM Drive, Momentum conservation, Newton's third law, ionized propellant, fluid, thrust, inelastic collision, enclosed chamber

\section{Introduction}

The publication of Roger Shawyer's EM Drive or electromagnetic drive along with apocryphal experimental confirmations has spotlighted the concept of propellant less propulsion. NASA scientist have tested the EM drive concept (among other institutions) and have found that the system can generate 1 Newton of thrust for every megawatt of power generated via electromagnetic waves within an enclosed chamber (Thompson, 2017). However, other experiments have shown that the validity of the EM drive concept is doubtful. Despite of how controversial the EM drive is, the prospect of a space propulsion system that is completely electrically dependent while expelling no propellant is quite stimulating to the development of innovative space propulsion research. The EM drive in junction with advances in ion propulsion are enriching to the field of space propulsion. The latest Advancements in ion propulsion have been made by the company Ad Astra founded by former astronaut Franklin Chang Diaz (Nadis, 2014). The company has developed ion rockets that have accelerated super-heated Argon gas up to velocities of 100,000 mph (Nadis, 2014). Thus evolutions in ion propulsion are underway and will reduce spacecraft transit times significantly. As is widely known in the propulsion engineering field, ion propulsion is fuel efficient, low powered, and has the potential to produce high velocities over time in space travel (NASA, 2016). An additional advantage to ion propulsion is that the majority of its power is produced by electricity (NASA, 2016). However, like conventional rockets, ion or plasma rockets will eventually deplete their propellant supply (in most cases Xenon and Argon gas) (Nadis, 2014) (NASA, 2016). This sets the premise of the assertion of this paper.

This paper introduces a theoretical propulsion concept which incorporates ion propulsion which similarly to the EM drive, does not lose propellant within an enclosed chamber. Thus, propulsion systems incorporating the theoretical propulsion concept generates thrust by circulating an ionized gas within an enclosed chamber as opposed to electromagnetic waves within a tapered enclosed chamber as with the EM Drive, hence the phrase within the title "a 
fluidic alternative to the EM drive". An arrangement of orthogonal fluid injectors (or nozzles) injects accelerated ionized gas into an enclosed chamber where electric forces generated by the chamber walls within the chamber govern the ionized gases. It will be mathematically shown that the ionized propellant injected by the orthogonal nozzles can generate thrust within the enclosed chamber and be recycled while obeying Newton's third law. Additionally, it will be shown that the law of conservation of momentum is obeyed within the theoretical concept. This theoretical propulsion concept is referred to as the orthogonal fluid flow propulsion concept or OFFPC.

Prior to introducing the core mathematics describing the physical attributes of the OFFPC, a one section description (section 1) of the EM drive and its core equations is given along with a brief comparison to the OFFPC. Section 2 presents a mathematical description and formulation of the core equations and mathematical expressions of the OFFPC, but more importantly, section 2 introduces the concept's obedience to (as previously expressed):

- Newton's third Law(with its relationship to momentum conservation)

- Aerodynamic momentum conservation in controlled volume systems

Thus after showing the important relationships to the aforementioned pivotal aspects of physics, derivations of the equations describing the electric forces generated by the chamber walls which govern the ionized propellant within the enclosed chamber are introduced (see sections 3 and 4). The OFFPC's ability to recycle its propellant will theoretically give an OFFPC propulsion system the ability to regenerate a small portion of its electric energy similarly to an alternator of an automobile. The OFFPC's ability to regenerate a portion of its energy is described and elucidated mathematically in section 5. One can impetuously conclude that the OFFPC assertion is that of a perpetual motion device as such criticisms faced (and continue to face) the EM drive. In response to this natural assumption, section 6 gives a description as to how the system expends, dissipates, and distributes energy like any real world system. Lastly, experimental values are applied to a hypothetical motor which incorporate the core equations and mathematical expressions of the OFFPC expressed throughout the paper to demonstrate and show performance figures that will convey the possibility of the theoretical propulsion concept in the conclusion.

\section{A Summary of the EM Drive}

As communicated in the introduction, the conception and introduction of the EM drive although controversial, has opened possibilities and awareness to new theoretical forms of propulsion such as the orthogonal fluid flow propulsion concept (OFFPC). Thus we give a mathematical summarization of Roger Shawyer's EM drive and a brief comparison to the OFFPC. To ensure the accuracy in the exposition of the EM drive, the following summary of the mathematics of the EM drive is meticulously extracted from Roger Shawyer's EM drive paper published by SPR LTD titled "A theory of microwave propulsion for space craft" (Shawyer, 2006). In commencing with a description of the EM drive, a magnetron emits microwave energy into the vacuum of a tapered cavity which constitutes an enclosed chamber to which one end has a greater area than the opposite end. The tapered cavity of the EM drive is depicted in figure 1 below (Shawyer, 2006).

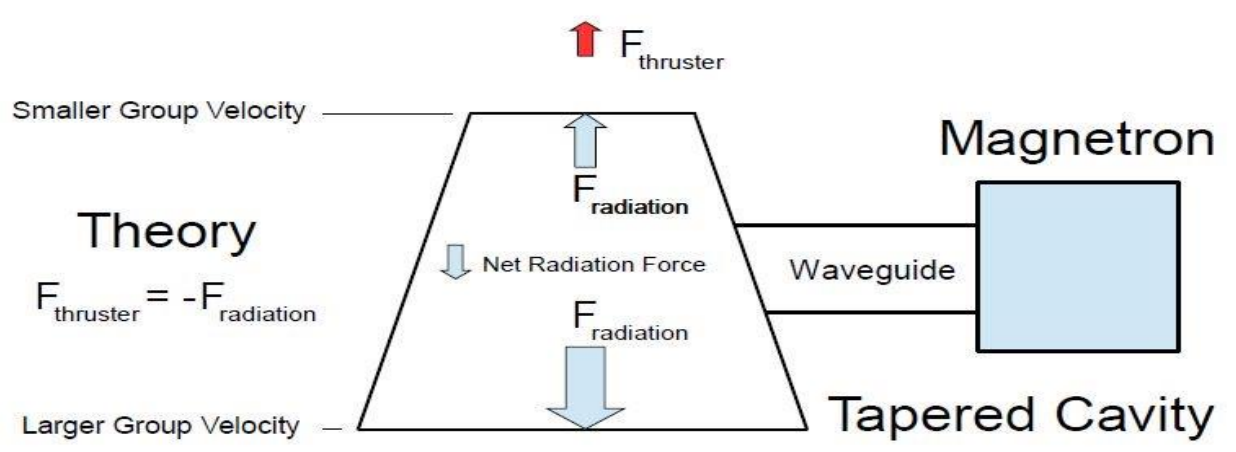

Figure 1. Image credit to https://hacked.com/wp-content/uploads/2015/07/em-drive-concept.jpg

The equation of force exerted by radiant energy emitted into the enclosed chamber is expressed such that (Shawyer, 2006):

$$
F_{\text {rad }}=\frac{2 P_{r a d}}{e}
$$

Where $F_{\text {rad }}$ (or $F_{\text {radiation }}$ as depicted in figure 1) is the force value exerted onto the structure of the enclosed chamber, 
$P_{\text {rad }}$ is the power associated with the radiant energy, and $c$ is the speed of light (Shawyer, 2006). The value of power $P_{\text {rad }}$ in Eq.1 can be mathematically expressed such that (Shawyer, 2006):

$$
P_{\text {rad }}=\text { nhfAc }
$$

Where $n$ is the number of photons per unit volume, $h$ is Planck's constant, $f$ is the frequency pertaining to the emitted electromagnetic microwaves, $A$ is the cross sectional area through which the electromagnetic energy propagates, and $c$ is the speed of light (Shawyer, 2006). The group velocity associated with the radiation is denoted $v_{g}$ (Shawyer, 2006). The group velocity $v_{g}$ is mathematically associated with the electromagnetic force $F_{\text {rad }}$ such that (Shawyer, 2006):

$$
F_{\text {rad }}=\frac{2 P_{\text {rad }}}{c}\left(\frac{v_{g}}{c}\right)
$$

The group velocity values associated with both ends of the tapered cavity which is an enclosed chamber are denoted $v_{g 1}$ and $v_{g 2}$ which correspond to the force values $F_{y a d 1}$ and $F_{r a d 2}$ (Shawyer, 2006). Thus the group velocity and force values of $v_{g 1}$ and $F_{\text {rad1 }}$ pertain to the larger end (of greater area) of the tapered chamber while group velocity $v_{g 2}$ and force $F_{\text {rad2 }}$ pertain to the smaller end (of lesser area) (Shawyer, 2006). The values of force $F_{\text {rad1 }}$ and $F_{\text {radz }}$ at both ends of the tapered cavity can be expressed such that (Shawyer, 2006):

$$
\begin{gathered}
F_{\text {rad1 }}=\frac{2 P_{\text {rad }}}{c}\left(\frac{v_{g 1}}{c}\right) \\
F_{\text {rad2 }}=\frac{2 P_{\text {rad }}}{c}\left(\frac{v_{g 2}}{c}\right)
\end{gathered}
$$

Additionally, in the case of a standing wave, the electromagnetic forces within the enclosed chamber (or tapered cavity) exerted along the tapered side walls continually cancel each other in opposite directions until the end wall plates (Shawyer, 2006). Therefore, the force values $F_{\text {rad1 }}$ and $F_{\text {rad2 } 2}$ at the end wall plates which pertain to the electromagnetic waves of radiation are maximum $\left(F_{\text {rad1 }}\right)$ and minimum $\left(F_{\text {rad2 }}\right)$ force values at the end plates (e.g. $F_{\text {rad1 } 1}>F_{\text {rad2 } 2}$ ) (Shawyer, 2006). Conclusively, the total thrust value $T$ (or $F_{\text {thruster }}$ as depicted in figure 1) is the difference between force values $F_{\text {rad1 }}$ and $F_{\text {rad2 }}$ that does not equal zero due to the inequality $F_{\text {rad1 }}>F_{\text {rad2 } 2}$ as expressed below (Shawyer, 2006).

$$
T=F_{\text {rad1 }}-F_{\text {rad } 2} \equiv \frac{2 P_{r a d}\left(v_{g 1}-v_{g 2}\right)}{c^{2}} \neq 0
$$

The group velocities $v_{g 1}$ and $v_{g 2}$ in Eq. 6 above are of the form (Shawyer, 2006):

$$
v_{g}=\frac{c}{\sqrt{\mu \varepsilon_{r}}} \frac{\lambda_{0}}{\lambda_{g 1}}
$$

Where $\mu$ and $e_{y}$ are constants pertaining to the dielectric material of the smaller end of the enclosed chamber which are set to unity $\left(\mu=e_{y}=1\right)$, the group velocities $v_{g 1}$ and $v_{g 2}$ are related to initial wavelength $\lambda_{0}$ and wavelengths $\lambda_{g 1}$ and $\lambda_{g 2}$ such that (Shawyer, 2006):

$$
\begin{gathered}
v_{g 1}=\frac{e \lambda_{0}}{\lambda_{g 1}} \\
v_{g 2}=\frac{c \lambda_{0}}{\lambda_{g 2}}
\end{gathered}
$$


Wavelengths $\lambda_{g_{1}}$ and $\lambda_{g_{2}}$ vary according to the cross sectional area of the tapered cavity, thus $\lambda_{g 1}<\lambda_{g_{2}}$ (Shawyer, 2006). This assertion leads to the force inequality $F_{\text {rad1 }}>F_{\text {rad2 } 2}$, which leads to the equation of thrust $T$ in terms of wavelength such that (Shawyer, 2006):

$$
T=F_{\text {rad1 }}-F_{\text {rad } 2} \equiv \frac{2 P_{\text {rad }}}{c}\left(\frac{\lambda_{0}}{\lambda_{g 1}}-\frac{\lambda_{0}}{\lambda_{g 2}}\right) \neq 0
$$

The radiation exerting pressure on the enclosed tapered chamber pertains to electromagnetic energy which implies that the group velocities must adhere to relativistic principles, thus group velocities $v_{g 1}$ and $v_{g 2}$ must obey Einstein's law of addition of velocities (Shawyer, 2006). Einstein's law of addition of velocities adheres to the form of Eq.11 below(Shawyer, 2006) .

$$
v=\frac{v_{1}+v_{2}}{1+\left(v_{1} v_{2}\right) / c^{2}}
$$

Applying Einstein's law of addition of velocities to the group velocities $v_{g 1}$ and $v_{g 2}$ of thrust equations $T$ of Eq.6 and 10, one obtains (Shawyer, 2006):

$$
T=\frac{2 P_{r a d} S_{0}}{c}\left(\frac{v_{g 1}-v_{g 2}}{1-\left(v_{g 1} v_{g 2}\right) / v c^{2}}\right) \equiv \frac{2 P_{r a d} S_{0}}{c}\left(\frac{\lambda_{0}}{\lambda_{g 1}}-\frac{\lambda_{0}}{\lambda_{g 2}}\right)
$$

Due to the incorporation of Einstein's law of addition of velocities, the thrust equation $T$ of Eq.12 has been corrected to include correction factor $S_{0}$ which has a value of Eq. 13 below (Shawyer, 2006).

$$
S_{0}=\left(1-\frac{\lambda_{0}{ }^{2}}{\lambda_{g 1} \lambda_{g 1}}\right)^{2}
$$

In deriving a final mathematical expression describing a thrust value of the EM drive, an additional correction must be implemented due to the fact that the smaller end of the tapered enclosed chamber is composed of a dielectric material to ensure a reflection free section of the chamber (Shawyer, 2006). Thus, the group velocity $v_{g 2}$ pertaining to the smaller end of the chamber is adjusted to describe the dielectric medium of the smaller end of the tapered enclosed chamber (Shawyer, 2006). Thus group velocity $v_{g 2}$ takes on a value such that (Shawyer, 2006):

$$
v_{g 2}=\frac{c \lambda_{d}}{\lambda_{g 3} \sqrt{\theta_{r}}}
$$

Where $\lambda_{d}$ is the wavelength of the unbounded dielectric medium, $\lambda_{g a}$ is the guide wavelength at the end plate of the dielectric section, and $e_{y}$ is a constant pertaining to the dielectric medium (Shawyer, 2006). Substituting the value of Eq.14 into the thrust equation of Eq.12, gives the final form of the EM drive thrust equation such that (Shawyer, 2006):

$$
T=\frac{2 P_{r a d} S_{0}}{c}\left(\frac{\lambda_{0}}{\lambda_{g 1}}-\frac{c \lambda_{d}}{\lambda_{g 3} \sqrt{E_{r}}}\right)
$$

At this juncture, the core function of the EM drive is well elucidated mathematically, thus we introduce and briefly compare the theoretical OFFPC propulsion system to the EM drive. As stated in the introduction, the OFFPC propulsion system uses an orthogonal arrangement of fluid streams consisting of ionized propellant which are injected into an enclosed chamber. The walls of the enclosed chamber generate electric fields whose forces (both repulsive and attractive) govern the ionized propellant. It is shown (in the following sections) that the ionized propellant is recycled and reused and thus is not lost to the vehicle while producing thrust similarly to the EM Drive. Hence, there is no loss or reduction in propellant mass, therefore, in a sense, the OFFPC is a form of propellant less propulsion and is electrically dependent as with the EM drive. In essence, the OFFPC propels itself off of its own internal atmosphere and can potentially produce higher thrust values as compared to the EM Drive. 


\section{The Orthogonal Fluid Flow Propulsion Concept (OFFPC)}

The core of the theoretical orthogonal fluid flow propulsion concept will be explicated in mathematical detail in section 2. In the exposition of the OFFPC, it is pivotal to the assertion to incorporate:

- Newton's third Law (with its relationship to momentum conservation)

- Aerodynamic momentum conservation in controlled volume systems

Thus the aforementioned points will be addressed shortly. Thus, prior to beginning a heuristic formulation of equations defining the OFFPC, it is important to emphasize the fact that all vehicle designs potentially featuring the orthogonal fluid-flow propulsion concept operate with orbital trajectories, thus all motion calculations of vehicles utilizing the OFFPC are to be applied at orbital altitudes and velocities or greater. From a practical and theoretical basis, the orthogonal fluid-flow propulsion concept will not produce enough power and thus thrust to achieve gravitational escape velocity. Hence, the system is suited for long duration space-flight similarly to conventional ion propulsion systems.

Therefore, in commencing with the formulation of the core equations and core concepts, a form of plasma (typically xenon gas) is injected into an enclosed chamber via three orthogonally oriented nozzles or injectors as shown in figure 2. The propellant is ionized by the conventional method of electron bombardment (for the purposes of this explanation) (NASA, 2016). Thus, the propellant plasma is injected with high-energy electrons using a discharge cathode (NASA, 2016). Initially, the propellant plasma has an even amount of positively charged atoms and negatively charged atoms prior to being bombarded by the electrons (hence a gas with a virtually neutral charge) (NASA, 2016). After the collision of high energy electrons, electrons are knocked free within the gas resulting in the propellant inheriting a dominantly positive charge (NASA, 2016). The positive charge of the ionized gas will be shown to be important to how the ionized propellant is governed within the enclosed chamber of the vehicle while generating propulsion or thrust via the OFFPC. High-strength magnets prevent electrons from freely reaching the discharge channel walls within the injector (NASA, 2016). This lengthens the time that electrons reside in the discharge chamber and increases the probability of an ionizing event(NASA, 2016).The positively charged ions of the propellant migrate toward grids that contain thousands of very precisely aligned holes (apertures) at the aft end of the ion thruster (i.e. the injector) (NASA, 2016). The first grid is a positively charged electrode (screen grid) (NASA, 2016). A very high positive voltage is applied to the screen grid, but it is configured to force the discharge plasma to reside at a high voltage (NASA, 2016). As ions pass between the grids, they are accelerated toward a negatively charged electrode (the accelerator grid) to very high speeds and expelled out of a nozzle into the enclosed chamber (NASA, 2016). This process transpires in three separate nozzles producing three individual ionized fluid flows which have dynamic pressure values of $P_{x}$ (on the x-axis), $P_{1 y}$ (on the y-axis), and $P_{2 y}$ (also on the y-axis opposing the fluid flow of pressure value $P_{1 y}$ ) as illustrated in figure 2. With the exception of the injected ionized propellant, the enclosed chamber is a vacuum. The injecting nozzles and thus dynamic pressure values $P_{1 y}$ and $P_{2 y}$ on the y-axis are orthogonal to dynamic pressure value $P_{x}$ and its nozzle on the $\mathrm{x}$-axis (which is the axis of travel of the vehicle) as shown in figure 2. Additionally, the positively charged ionized propellant fluid is kept from contacting the chamber side walls due to the repulsive force $\left(F_{\mathscr{S W}}\right)$ of the positively charged chamber side wall (which will be defined in mathematical detail in the section 4). Thus, due to positively charged repulsive force $\left(F_{\mathscr{S W}}\right)$, the positively charged ionized propellant is suspended or confined to the center of the open volume of the enclosed chamber along the $\mathrm{x}$-axis as shown in figure 2.The chamber side walls are the lower plate surfaces of a capacitor which conduct electrons away from the chamber side wall surface generating a positive charge and a repulsive force to the positively charged ionized propellant which prevents the ionized propellant from exerting on the structure of the enclosed chamber via the chamber side wall. Resultantly, the ionized propellant makes no exertion on the $\mathrm{x}$-axis in either direction via the side chamber wall. As shown in figure 2 , the negatively charged electric force $F_{\text {ema }}$ (defined in detail in section 3) will adhere or trap the ionized propellant to the forward chamber wall perpendicular to the direction and axis of travel (the x-axis) to create an inelastic collision (Young \& Freedman, 2004). 


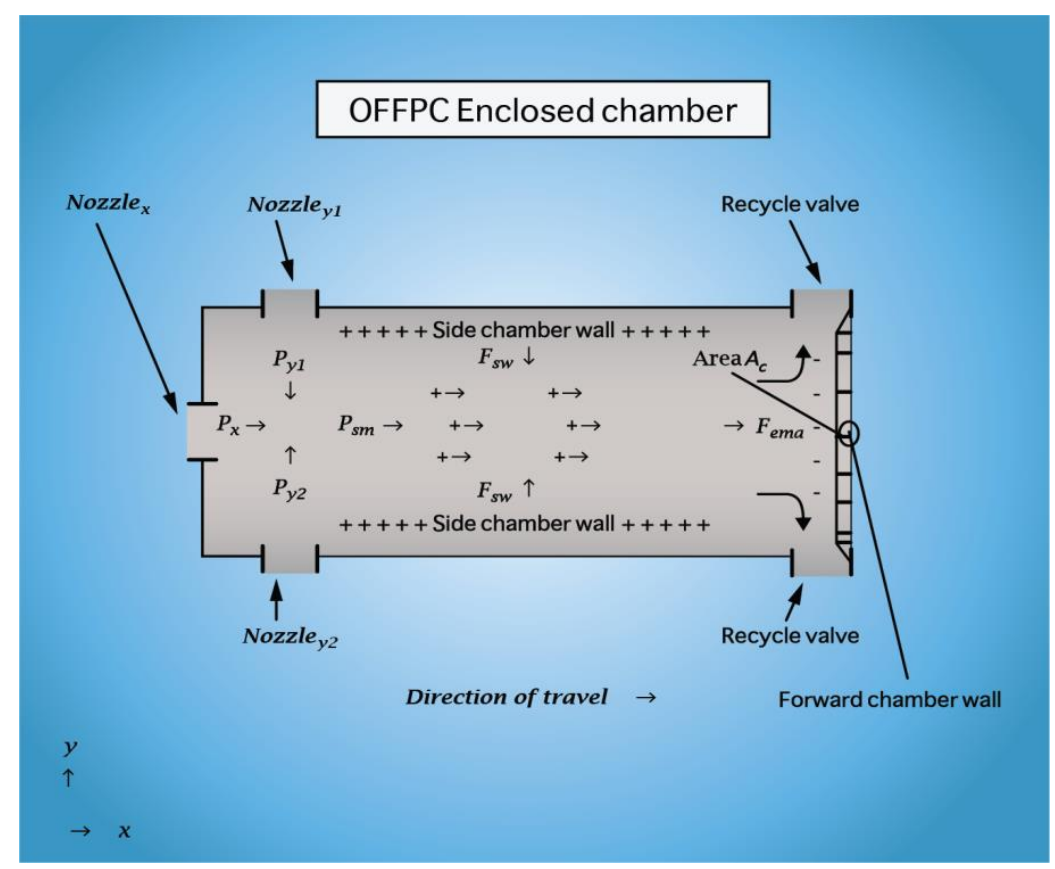

Figure 2. The OFFPC enclosed chamber

The ionized propellant is a fluid, thus, the description of the ionized propellant within the enclosed chamber is required to be expressed in terms of pressure and energy. In fluid mechanics, pressure $P$ relates to energy via the pressure/energy density equation of (Hyper Physics, 2017) (Young\& Freedman, 2004):

$$
P=\frac{W}{V_{c}}=\frac{E}{V_{c}}
$$

Where $W$ denotes work or energy $E$ per enclosed chamber volume $V_{c}$, therefore pressure $P$ also constitutes the energy density of the fluid i.e. the ionized propellant (Hyper Physics, 2017) (Young \& Freedman,2004). Thus, dynamic pressure $P$ relates to kinetic energy $K\left(K=m(v)^{2} / 2\right)$ such that (Young \&Freedman, 2004):

$$
P=\frac{K}{V_{c}}=\frac{\rho(v)^{2}}{2}
$$

Where $v$ denotes the fluid velocity and $\rho$ denotes the fluid density $\left(\rho=m / V_{c}\right)$. Hence relating this to the OFFPC, the orthogonal fluid flows of dynamic pressure values $P_{x}, P_{2 y}$, and $P_{1 y}$ produced by the three nozzles relate to kinetic energy values $K_{x}, K_{1 y}$, and $K_{2 y}$ per volume $V_{c}$ such that:

$$
\begin{aligned}
& P_{x}=\frac{K_{x}}{V_{c}}=\frac{\rho_{x}\left(V_{x}\right)^{2}}{2} \\
& P_{1 y}=\frac{K_{1 y}}{V_{c}}=\frac{\rho_{1 y}\left(V_{1 y}\right)^{2}}{2} \\
& P_{2 y}=\frac{K_{2 y}}{V_{c}}=\frac{\rho_{2 y}\left(V_{2 y}\right)^{2}}{2}
\end{aligned}
$$

Where $v_{x}, v_{1 y}$, and $v_{2 y}$ are the corresponding fluid velocities for each fluid flow. It must be noted that $\mathrm{y}$-axis dynamic pressure values $P_{1 y}$ and $P_{2 y}$ are equal in magnitude $\left(\left|P_{1 y}\right|=\left|P_{2 y}\right|\right)$ as expressed below. 


$$
P_{1 y}=P_{2 y}
$$

Additionally, the magnitude of the dynamic pressure value on the x-axis of dynamic pressure value $P_{x}$ must be greater than the combined sum of dynamic pressure values $P_{1 y}$ and $P_{2 y}$ oriented on the perpendicular axis (or y-axis) as conveyed by the inequality below.

$$
P_{x}>P_{1 y}+P_{2 y}
$$

The inequality above ensures that the more powerful fluid stream of dynamic pressure $P_{x}$ on the x-axis diverts the direction of the dynamic pressure of orthogonal streams $P_{1 y}$ and $P_{2 y}$ into the direction of the stream of dynamic $P_{x}$ towards the forward chamber wall; i.e. all of the fluid streams travel in the same direction on the $\mathrm{x}$-axis after the collision of all of the streams of ionized propellant. The mass values of $m_{x}, m_{1 y}$, and $m_{2 y}$ corresponding to density values $\rho_{x}, \rho_{1 y}$, and $\rho_{2 y}$ produced by the three orthogonal nozzles are the mass values within the enclosed chamber over a time span $\Delta t$. Therefore, each mass value $m_{x}, m_{1 y}$, and $m_{2 y}$ can be presented as the product of the mass per time rate $\dot{m}(\dot{m}=m / \Delta t)$ and timespan $\Delta t$ as expressed below (Benson, 2013).

$$
m=\dot{m} \Delta t=\left(\frac{m}{\Delta t}\right) \Delta t \quad \quad \quad \text { where } \quad \dot{m}=\frac{m}{\Delta t}
$$

Thus, the values of fluid densities on the $\mathrm{x}$-axis and the y-axis of $\rho_{x}, \rho_{1 y}$, and $\rho_{2 y}$ within a chamber volume $V_{c}$ are expressed such that:

$$
\begin{gathered}
\rho_{x}=\frac{m_{x} \Delta t}{v_{c}}=\frac{m_{x}}{v_{c}} \\
\rho_{1 y}=\frac{m_{1 y} \Delta t}{v_{c}}=\frac{m_{1 y}}{v_{c}} \\
\rho_{2 y}=\frac{m_{2 y} \Delta t}{v_{c}}=\frac{m_{2 y}}{v_{c}}
\end{gathered}
$$

Therefore, we have the propellant density values produced by each nozzle within a timespan $\Delta t$. The total pressure/energy of the colloidal mass of ionized propellant which is confined to the center of the enclosed chamber via the repulsive force $F_{S W}$ (to be defined in section 4) pertaining to the positive charge of the side chamber wall as seen in figure 2 will be referred to as the suspended mass within the text. Thus, due to the energy density equation of Eq.16 $(P=E / V)$, the total kinetic energy of the suspended propellant mass denoted $K_{s m}$ (the subscript $s m$ referring to "suspended mass") associated with all three nozzles and thus fluid flows is the sum of the kinetic energy values of $K_{x}$, $K_{1 y}$, and $K_{2 y}$ such that (Young \& Freedman, 2004):

$$
K_{s m}=V_{c} P_{s m}=K_{x}+K_{1 y}+K_{2 y}
$$

Where $P_{s m}$ denotes the dynamic pressure of the suspended mass of all three fluid flows and $V_{c}$ denotes the volume of the enclosed chamber, Eq.27 can alternatively be expressed as:

$$
K_{g m}=\frac{V_{c} \rho_{x}\left(V_{x}\right)^{2}}{2}+V_{c}\left(\frac{\rho_{1 y\left(V_{1 y}\right)^{2}}}{2}+\frac{\rho_{2 y\left(V_{2 y}\right)^{2}}}{2}\right)
$$

Hence, the dynamic pressure or energy density of the suspended mass of the ionized propellant $P_{\mathscr{s m}}$ is given such that:

$$
P_{\mathrm{gm}}=\frac{K_{s m}}{V_{c}}=P_{x}+P_{1 y}+P_{2 y}
$$


Or alternatively, in terms of energy density, dynamic pressure $P_{g m}$ can be expressed in terms of kinetic energy per volume such that:

$$
P_{s m}=\frac{K_{x}}{V_{c}}+\frac{R_{1 y}}{V_{c}}+\frac{R_{2 y}}{V_{c}}
$$

Hence, the dynamic pressure of all three fluid flows can alternatively be expressed as shown below.

$$
P_{g m}=\frac{\rho_{x}\left(\mathrm{~N}_{x}\right)^{2}}{2}+\left(\frac{\rho_{1 y}\left(\mathrm{~N}_{1 y}\right)^{2}}{2}+\frac{\rho_{2 y}\left(\mathrm{~N}_{2 y}\right)^{2}}{2}\right)
$$

The combined pressure all three fluid flows confined to the center of the chamber can be set equal to a single term such that :

$$
P_{s m}=\frac{\rho_{s m}\left(V_{s m}\right)^{2}}{2}
$$

The fluid velocity $v_{\mathrm{gm}}$ is the resultant velocity pertaining to the collision of all three propellant fluid flows confined to the center of the enclosed chamber via electromagnetic force; i.e. fluid velocity $v_{\mathrm{sm}}$ is the combined fluid velocity of the suspended propellant mass. Fluid density $\rho_{s m}$ is the resultant density of all three propellant fluid flows. Fluid density $\rho_{s m}$ per chamber volume $V_{c}$ takes on a value such that:

$$
\rho_{s m}=\frac{m_{x}+m_{1 y}+m_{2 y}}{V_{c}}
$$

Thus Eq. 29-31 can then be re-expressed such that:

$$
\frac{\rho_{s m}\left(V_{m m}\right)^{2}}{2}=\frac{\rho_{x}\left(\mathbb{V}_{x}\right)^{2}}{2}+\left(\frac{\rho_{1 y}\left(\mathrm{~V}_{1 y}\right)^{2}}{2}+\frac{\rho_{2 y}\left(\mathrm{~V}_{2 y}\right)^{2}}{2}\right) \equiv P_{g m}
$$

Therefore, solving Eq. 34 for velocity $v_{s m}$, the velocity of the suspended mass i.e. velocity $v_{s m}$ takes on a value such that:

$$
V_{s m}=\left[\frac{2}{\rho_{s m}}\left[\frac{\rho_{x}\left(V_{x}\right)^{2}}{2}+\left(\frac{\rho_{1 y}\left(V_{1 y}\right)^{2}}{2}+\frac{\rho_{2 y}\left(V_{2 y}\right)^{2}}{2}\right)\right]\right]^{1 / 2} \equiv \sqrt{\frac{2 \rho_{s m}}{\rho_{s m}}}
$$

Force relates to pressure and area via the classical formula force $=$ pressure $\times$ area (Young \& Freedman, 2004). Thus, as the force exerted by the suspended mass of the fluid propellant on the forward chamber wall of area $A_{\varepsilon}$ (as depicted in figure 2), force $F_{g m}$ is exerted onto the forward chamber wall. Aerodynamic force $F_{s m}$ which is normal to the forward chamber wall takes on a value such that:

$$
F_{g m}=A_{c} P_{s m}=A_{c} P_{x}+A_{c} P_{1 y}+A_{c} P_{2 y}
$$

Suspended mass force $F_{g m}$ is alternatively expressed such that:

$$
F_{s m}=\frac{A_{c} \rho_{x}\left(N_{x}\right)^{2}}{2}+A_{c}\left(\frac{\rho_{1 y}\left(v_{1 y}\right)^{2}}{2}+\frac{\rho_{2 y}\left(N_{2 y}\right)^{2}}{2}\right)
$$

Newton's third law within the OFFPC: The establishment of Eq.37 above allows us to begin defining the OFFPC relationship to Newton's third law and the formulation of the equation of thrust pertaining to the enclosed chamber. Due to Newton's third law $\left(\sum F_{x}=F_{x 0}-F_{x}=0\right.$ ), the x-axis component of force $F_{x}$ (i.e. the term $A_{c} P_{x}$ or $A_{c} \rho_{x}\left(v_{x}\right)^{2} / 2$ in Eq. 36-37) within the suspended mass force $F_{s m}$ exerted on the forward chamber wall of area $A_{c}$ must equal the force exerted by the nozzle producing dynamic pressure $P_{x}$ located on the opposite end of the enclosed chamber 
(Young \& Freedman, 2004). The x-axis force value $F_{x}$ (i.e. $F_{x}=A_{e} P_{x}$ ) within suspended mass force value $F_{g m}$ of Eq.37 is highlighted in the equation below.

$$
F_{s m}=F_{x}+A_{c}\left(\frac{\rho_{1 y}\left(\mathbb{v}_{1 y}\right)^{2}}{2}+\frac{\rho_{2 y}\left(\mathbb{v}_{2 y}\right)^{2}}{2}\right)
$$

Therefore, $\mathrm{x}$-axis force value $F_{x}$ takes on a value such that (Young \& Freedman, 2004):

$$
F_{x}=\frac{A_{c} \rho_{x}\left(V_{x}\right)^{2}}{2}
$$

Hence, due to Newton's third law, the force value denoted $F_{x 0}$ exerted by the x-axis nozzle on the end opposite to the forward chamber wall must be equal to the x-axis term of force $F_{x}$ of Eq. $39\left(F_{x 0}=F_{x}\right)$ within suspended mass force $F_{s m}$ exerted on the forward chamber wall, this is mathematically expressed such that (Young \& Freedman, 2004):

$$
\sum F_{x}=F_{x 0}-F_{x}=0 \quad \rightarrow \quad F_{x 0}=F_{x}
$$

The nozzle on the $\mathrm{x}$-axis which is the axis of travel generates a force $F_{x 0}$ on one side of the enclosed chamber. In obtaining an expression for x-axis nozzle force $F_{x 0}$, we acknowledge the general thrust equation denoted $F_{T}$ expressed below (Benson, 2013):

$$
F_{T}=\left(v_{f}-v_{\mathrm{i}}\right) \dot{m}+\left(P_{f}-P_{\mathrm{i}}\right) A
$$

Where $v_{\mathrm{m}}\left(v_{\mathrm{i}}, v_{f}\right)$ is the fluid velocity, $P_{\mathrm{m}}\left(P_{\mathrm{i}}, P_{f}\right)$ denotes pressure, $\dot{m}$ denotes mass per time such that $\dot{m}=m / \Delta t$ (of Eq.23), and area $A$ is the nozzle opening area; force $F_{x 0}$ takes on the form of the thrust equation of Eq.41 (i.e. $\left.F_{x 0}=F_{T}\right)$ (Benson, 2013). Where the initial values of velocities and pressure values are zero $\left(v_{i}=0\right.$ and $P_{i}=0$ )and the final velocity is $v_{f}=v_{x}$ and the final pressure is $P_{f}=P_{x}$ within the nozzle after ionization and after acceleration of the ionized propellant. Therefore, $\mathrm{x}$-axis nozzle force $F_{x 0}$ takes on a value such that:

$$
F_{x 0}=v_{x} \dot{m}_{x}+A_{x} P_{x} \equiv v_{x} \dot{m}_{x}+\frac{A_{x} \rho_{x}\left(v_{x}\right)^{2}}{2}
$$

As previously expressed, the force value exerted by the nozzle denoted $F_{x 0}$ must equal the force exerted by force $F_{x}\left(F_{x}=F_{x Q}\right)$ along the x-axis in accordance to Newton's third law (Young \& Freedman, 2004), this equivalence in force values can be expressed as Eq.43 below.

$$
\frac{A_{c} \rho_{x}\left(W_{x}\right)^{2}}{2}=v_{x} \dot{m}_{x}+\frac{A_{x} \rho_{x}\left(V_{x}\right)^{2}}{2}
$$

In satisfying Eq. 43 , we solve for nozzle opening area $A_{x}$, thus giving a value such that:

$$
A_{x}=A_{c}-\frac{2 w_{x}}{\rho_{x} v_{x}}
$$

With the condition of the inequality of:

$$
A_{c}>\frac{2 m_{x}}{\rho_{x} v_{x}}
$$

This is a prelude to the formulation an equation of total force or thrust exerted on the structure of the enclosed chamber and thus vehicle along the axis of travel (which is the x-axis). Total force or thrust exerted on the entire enclosed chamber (and thus vehicle) denoted $F_{X^{\prime}}$ along the axis of travel (the x-axis) is the difference between suspended propellant mass force $F_{g m}$ and x-axis nozzle force $F_{x 0}$ on opposite ends of the enclosed chamber as shown below. 


$$
F_{x^{\prime}}=F_{s m}-F_{x 0}
$$

This can alternatively be expressed such that:

$$
F_{x^{\prime}}=\left(\frac{A_{c} \rho_{x}\left(V_{x}\right)^{2}}{2}+A_{c}\left(\frac{\rho_{1 y}\left(V_{1 y}\right)^{2}}{2}+\frac{\rho_{2 y}\left(V_{2 y}\right)^{2}}{2}\right)\right)-\left(v_{x} \dot{m}_{x}+\frac{A_{x} \rho_{x}\left(V_{x}\right)^{2}}{2}\right)
$$

Eq.47 can be rearranged such that:

$$
F_{x^{\prime}}=\left(\left(\frac{A_{c} \rho_{x}\left(v_{x}\right)^{2}}{2}-\left(v_{x} \dot{m}_{x}+\frac{A_{x} \rho_{x}\left(v_{x}\right)^{2}}{2}\right)\right)+A_{c}\left(\frac{\rho_{1 y}\left(\mathbb{v}_{1 y}\right)^{2}}{2}+\frac{\rho_{2 y}\left(v_{2 y}\right)^{2}}{2}\right)\right)
$$

Where (for $A_{x}=A_{c}-2 \tilde{m}_{x} / P_{x} v_{x}$ of Eq.44) due to Newton's third Law $\left(\sum F_{x}=F_{x 0}-F_{x}=0\right.$ ), the x-components of fluid force highlighted above cancel out or equal zero as conveyed in Eq.49 below.

$$
\left(\frac{A_{c} \rho_{x}\left(\mathbb{N}_{x}\right)^{2}}{2}-\left(v_{x} \dot{m}_{x}+\frac{A_{x} \rho_{x}\left(\mathbb{N}_{x}\right)^{2}}{2}\right)\right)=0
$$

An important consideration is that although Eq.49 above is true for the value of nozzle area $A_{x}$ for the purpose of this formulation, Newton's third law must be obeyed for any variable. This implies that if Eq.49 is not true for nozzle area $A_{x}$ above, then Eq.49 must hold true for the variables of area $A_{\varepsilon}$, velocity $v_{x}$, or density $p_{x}$ thus satisfying Newton's third law.

An important question one must ask in reference to Eq.49 is what occurs to the value of kinetic energy $K_{x}\left(K_{x}=V_{c} P_{x}\right)$ of the ionized propellant on the $\mathrm{x}$-axis when the corresponding $\mathrm{x}$-axis dynamic pressure values cancel out due to Newton's third law. One can rhetorically ask if the law of conservation of energy has been violated. This query is addressed in section 6 in detail, however, the simplest explanation to this question is that the force and thus energy is absorbed by the structure of the enclosed chamber and converted to thermal energy due to the fact that the ionized propellant is temporarily trapped to forward chamber wall via electric force $\left(F_{\text {ema }}\right)$ as will be shown shortly. Therefore, in continuing the formulations; due to Eq.49, force $F_{x^{\prime}}$ of Eq. 48 can be expressed such that:

$$
F_{z^{\prime}}=\left((0)+A_{c}\left(\frac{\rho_{1 y\left(\mathbb{N}_{1 y}\right)^{2}}}{2}+\frac{\rho_{2 y}\left(\mathbb{N}_{2 y}\right)^{2}}{2}\right)\right)
$$

Which reduces to:

$$
F_{x^{\prime}}=A_{c}\left(\frac{\rho_{1 y\left(\mathbb{N}_{1 y}\right)^{2}}}{2}+\frac{\rho_{2 y}\left(\mathbb{v}_{2 y}\right)^{2}}{2}\right) \equiv A_{c} P_{1 y}+A_{c} P_{2 y}
$$

As previously expressed, due to the required inequality of $P_{x}>P_{2 y}+P_{1 y}$ of Eq.22, the direction of dynamic pressure values $P_{1 y}$ and $P_{2 y}$ is diverted along the x-axis toward the forward chamber wall upon the collision with the x-axis stream of dynamic pressure $P_{x}$. Conclusively, total thrust $F_{X^{\prime}}$ has a nonzero value showing that the OFFPC does in theory generate thrust as conveyed below.

$$
F_{X^{\prime}}=\left(\frac{A_{c} \rho_{x}\left(v_{x}\right)^{2}}{2}+A_{c}\left(\frac{\rho_{1 y\left(\mathbb{N}_{1 y}\right)^{2}}}{2}+\frac{\rho_{2 y\left(\mathbb{V}_{2 y}\right)^{2}}}{2}\right)\right)-\left(v_{x} \dot{m}_{x}+\frac{A_{x} \rho_{x}\left(\mathbb{V}_{x}\right)^{2}}{2}\right) \neq 0
$$

This can be congruently expressed as:

$$
F_{x^{\prime}}=A_{c}\left(\frac{\rho_{1 y}\left(\mathbb{1}_{1 y}\right)^{2}}{2}+\frac{\rho_{2 y}\left(\mathrm{v}_{2 y}\right)^{2}}{2}\right) \neq 0
$$

Essentially, the diverted pressure and energy of the y-axis dynamic pressure values $P_{1 y}$, and $P_{2 y}$ after the collision of 
fluid streams generates thrust and thus force value $F_{X^{\prime}}$ on the x-axis. It will be shown that the definition of momentum conservation in the OFFPC is highly dependent on the inelastic collision of the ionized propellant with the forward chamber wall. The generation of thrust is also dependent on the inelastic collision of the ionized propellant with the forward chamber wall. Therefore, we begin with the description of the ionized propellant being trapped along the forward chamber wall. The part of the sequence pertaining to the movement of ionized propellant within the enclosed chamber that allow the ionized propellant to exit or egress through the recycle values (shown in figure 2) while transferring its kinetic energy to the entire structure of the enclosed chamber and hence vehicle to produce thrust will now be conveyed. Once the ionized propellant collides with the forward chamber wall, an electric force temporarily (within time span $\Delta t$ ) traps the propellant to the forward chamber wall causing an inelastic collision (Young \& Freedman,2004). Therefore, the electromagnetic adhesive force $F_{\text {ema }}$ adheres or temporarily (within time span $\Delta t$ ) bonds the ionized propellant to the structure of the enclosed chamber and hence the entire vehicle. Figure 2 depicts the forward chamber wall which has a surface area $A_{c}$ and generates an electric force value of $F_{\text {ema }}$ (to be defined in the next section). The magnitude of negatively charged electric force value $F_{e m a}$ is required to be much greater than the magnitude of the suspended mass fluid force value $F_{g m}$, which is the combined force exerted on area $A_{\varepsilon}$ of the forward chamber wall by the ionized propellant.

$$
\left|F_{e m a}\right|>\left|F_{s m}\right|
$$

This can alternatively be expressed such that:

$$
\left|F_{e m a}\right|>>\left|\frac{A_{\varepsilon} \rho_{x}\left(D_{x}\right)^{2}}{2}+A_{c}\left(\frac{\rho_{1 y}\left(v_{1 y}\right)^{2}}{2}+\frac{\rho_{2 y}\left(v_{2 y}\right)^{2}}{2}\right)\right|
$$

The inequalities Eq. 54-55 is a required condition in order to sufficiently trap the charged particles of the ionized propellant in order to produce an inelastic collision (Young \& Freedman, 2004). It is Important to recognize the fact that the velocity of the ionized propellant becomes zero relative to the motion of the entire vehicle as the ionized propellant is trapped along the forward chamber wall via the negatively charged electric force $F_{e m a}$ generated by the forward chamber wall. This is analogous to a child running and jumping onto a scooter that is not in motion, the energy or momentum of the child is transferred into the motion of both the child and the scooter. Therefore, both the mass of the scooter and the child gain momentum and kinetic energy as a single object $\left(P_{\text {both }}=v(\right.$ mass of child + mass of scooter $\left.)\right)$ at velocity $v$ (Young \& Freedman, 2004). Thus, the electric force $F_{e m a}$ is representative of the gripping of the hands and shoes to which keep the child attached to the scooter as both move forward. The momentum value $p_{v}$ is the value of momentum enacted on the entire vehicle. Thus velocity value $v_{D}$ is the resultant velocity enacted on the entire vehicle corresponding to momentum value $p_{D}$. Momentum $p_{D}$ has a value such that (Young \& Freedman, 2004):

$$
p_{v}=v_{v}\left(m_{v}+m_{x}+m_{1 y}+m_{2 y}\right)
$$

Where mass $m_{v}$ is the mass value of the entire vehicle (including the enclosed chamber), electric force $F_{\text {ema }}$ allows the sum of all mass values $m_{v}+m_{x}+m_{1 y}+m_{2 y}$ over area $A_{c}$ of the forward chamber wall and hence the temporary adhesion (or bonding) of propellant mass to the mass of the vehicle $m_{v}$. Momentum $p_{v}$ of Eq.56 is set equal to momentum $p_{x}$ shown below.

$$
p_{v}=p_{x^{\prime}}
$$

Where momentum $p_{x^{\prime}}$ is equal to the product of time span $\Delta t$ and force $F_{x^{\prime}}$ of Eq.53 or $51\left(p_{x^{\prime}}=\Delta t F_{x^{\prime}}\right)$, momentum $p_{x^{\prime}}$ is the amount of momentum exerted on the entire vehicle by the ionized propellant and the resultant momentum is $p_{v}$ which corresponds to the resultant vehicle velocity $v_{b}$. Hence, the momentum $p_{x^{\prime}}$ constitutes the ionized propellant's impact and adhesion to the structure which produces and equal momentum of motion $p_{v}$ (i.e. $p_{v}=p_{x^{\prime}}$ of Eq.57) Conclusively, we see that momentum is conserved within the vehicle $\left(p_{v}-p_{x^{\prime}}=0\right)$. The fluids or propellant in the ionized propellant within the enclosed chamber possessing a closed volume will also show momentum conservation as will be shown shortly (See Eq.64). Therefore, Eq.57 can alternatively be expressed such that:

$$
v_{v}\left(m_{v}+m_{x}+m_{1 y}+m_{2 y}\right)=\Delta t\left(A_{c}\left(\frac{\rho_{1 y}\left(\nu_{1 y}\right)^{2}}{2}+\frac{\rho_{2 y}\left(\omega_{2 y}\right)^{2}}{2}\right)\right)
$$


Thus, the resultant vehicle velocity $v_{D}$ takes on a value such that:

$$
v_{v}=\frac{\Delta t}{\left(m_{v}+m_{x}+m_{1 y}+m_{2 y}\right)}\left[\left(A_{c}\left(\frac{\rho_{1 y}\left(v_{1 y}\right)^{2}}{2}+\frac{\rho_{2 y}\left(v_{2 y}\right)^{2}}{2}\right)\right)\right]
$$

The force of thrust $F_{\text {thrust }}$ enacted on the entire vehicle can be presented in the form of Newton's force equation ( $\mathrm{F}=\mathrm{ma}$ ) such that (Young \& Freedman, 2004):

$$
F_{\text {thrust }}=a_{v}\left(m_{v}+m_{x}+m_{1 y}+m_{2 y}\right)
$$

Where $a_{v}$ is the acceleration enacted on the entire vehicle, the force of thrust $F_{\text {thrust }}$ is set equal to force value $F_{X^{\prime}}$ of Eq.51 such that:

$$
F_{\text {thrust }}=F_{x^{\prime}}
$$

This can be alternatively expressed such that:

$$
a_{v}\left(m_{v}+m_{x}+m_{1 y}+m_{2 y}\right)=\left(A_{c}\left(\frac{\rho_{1 y}\left(v_{1 y}\right)^{2}}{2}+\frac{\rho_{2 y}\left(v_{2 y}\right)^{2}}{2}\right)\right)
$$

Hence, the propulsion system of the enclosed chamber will generate an acceleration value such that:

$$
\left.a_{v}=\frac{1}{\left(m_{v}+m_{x}+m_{1 y}+m_{2 y}\right)}\right]\left[\left(A_{c}\left(\frac{\rho_{1 y}\left(v_{1 y}\right)^{2}}{2}+\frac{\rho_{2 y}\left(v_{2 y}\right)^{2}}{2}\right)\right)\right]
$$

After temporarily bonding to the vehicle structure while transferring its energy, the ionized propellant mass then egresses from the chamber via the recycle valves located alongside the forward chamber wall. Thus Eq.63 completes a sufficient description of thrust and acceleration values exerted on the vehicle by the OFFPC. At this juncture, we are prepared to define momentum conservation within the OFFPC.

Momentum conservation in a controlled volume systems of the OFFPC

At this juncture, the conservation of momentum issue can be addressed, more specifically, the conservation of momentum in an aerodynamic controlled volume (or fix volume) system. Aerodynamically, the OFFPC is a controlled volume system, hence, momentum conservation is mathematically described by the equation of (Penn state University, 2017):

$$
\sum F_{x}=\frac{d}{d t} \int \rho v d V+\oint \rho v(v \bullet n) d A
$$

Where $F_{x}$ denotes aerodynamic force along the axis of travel, $\rho$ the corresponding fluid density, $v$ is the velocity pertaining to the acceleration (or force/momentum) exerted by the fluid, $d V$ is the volume element, $d A$ pertains to the instantaneous change in area, and $n$ denotes the normal force which is perpendicular the cross sectional area of efflux (which is the area $A_{c}$ of the forward chamber wall) (Penn state University, 2017). Due to the positively charged repulsive forces of the chamber side walls $\left(F_{G V}\right)$ of the OFFPC enclosed chamber, the ionized propellant does not come into contact with the chamber walls until the fluid comes into contact with the forward chamber wall, thus, the viscosity of the propellant does factor into calculations of Eq.64(Penn state University,2017). Additionally, it has been stated that propulsion systems incorporating the OFFPC are operated at orbital altitudes and beyond, thus, the effects of gravity on the ionized propellant within the enclosed chamber are nonexistent and thus excluded from the controlled volume momentum conservation equation of Eq. 64. The total aerodynamic force of the ionized propellant within the OFFPC enclosed chamber force is fluid force $F_{s m}$ of Eq.37. Fluid force $F_{s m}$ can be expressed in terms of Newton's force equation $(f=m a)$, thus force $F_{s m}$ is set equal to the product of a corresponding acceleration $a_{g m}$ and an arbitrary mass value $m$ (e.g. $F_{s m}=m a_{s m}$ ). The momentum value associated with aerodynamic force $F_{s m}$ denoted $p_{s m}$ is mathematically expressed in terms of time interval $\Delta t$ (such that: $p_{s m}=\Delta t F_{s m} \equiv \Delta t m a_{s m}$ ) (Young \& Freedman, 2004). Thus, the total force $\sum F_{x}$ of Eq. 64 can be expressed in terms of a differential such that: 


$$
\sum F_{x}=\frac{d p_{s m}}{d \Delta t} \equiv F_{g m}
$$

Therefore Eq.65 above is substituted into Eq.64 and density $\rho$ is set equal to $\rho_{s m}$ of Eq.33 $\left(\rho=\rho_{s m}\right)$, yielding the momentum conservation equation for a controlled volume in terms of the OFFPC such that:

$$
\frac{d p_{s m}}{d \Delta t}=\frac{d}{d \Delta t} \int \rho_{s m} v d V+\oint \rho_{s m} v(v \bullet n) d A
$$

The first term of Eq. 66 highlighted in yellow above, can be expressed such that (Penn state University, 2017):

$$
\frac{d}{d \Delta t} \int \rho_{s m} v d V=\rho_{s m} \frac{d v}{d \Delta t} V_{c}=m_{s m} \frac{d v}{d \Delta t}
$$

Where $V_{c}$ denotes the enclosed chamber volume of the OFFPC and mass $m_{g m}$ is the total propellant mass $\left(m_{s m}=m_{x}+m_{1 y}+m_{2 y}\right.$ for $\left.\rho_{s m}=m_{s m} / V_{c}\right)$ within time interval, evaluating the integral in respect to volume $(d V)$ gives the value of the integral as shown in Eq.67 above. The latter term (and thus integral) of Eq.66 has a value such that (Penn state University, 2017 ):

$$
\oint \rho_{s m} v(v \bullet n) d A=v_{f} A_{f} \rho_{s m}-v_{i} A_{i} \rho_{s m}
$$

Where $v_{\mathrm{i}}$ and $v_{f}$ are the initial and final fluid velocities, and $A_{\mathrm{i}}$ and $A_{f}$ are the initial and final cross sectional areas of the fluid flow which pertain to the continuity equation and thus the enclosed chamber (Penn state University, 2017). The corresponding density value is that of $\rho_{s m}$ of Eq.33; recall that velocity $v$ is the velocity pertaining to the acceleration value $a_{g m}$ and thus the aerodynamic force/momentum $\left(F_{g m}\right.$ or $p_{g m}$ ) exerted by the fluid (or ionized propellant) (Penn state University, 2017). Conversely, the velocities of $v_{i}$ and $v_{f}$ of Eq. 68 pertain to the energy density/ pressure of the ionized propellant (as opposed to velocity v). As previously expressed, when the ionized propellant comes into contact with the forward chamber wall, the propellant is trapped by the force $F_{\text {ema }}$ generated by the forward chamber wall producing an inelastic collision (Penn state University, 2017). Therefore, the initial velocity $v_{\mathrm{i}}$ is equal to the fluid velocity of $v_{s m}$ of Eq. $35\left(v_{i}=v_{s m}\right)$ which is the velocity correlating to pressure $P_{s m}$, and the final velocity of the fluid $v_{f}$ is equal to zero $\left(v_{f}=0\right)$ due to the stoppage of the fluid on the forward chamber wall (Penn state University, 2017). As implied by figure 2, there is no change in the cross sectional area of the enclosed chamber, therefore, the initial and final cross sectional areas of $A_{\mathrm{i}}$ and $A_{f}$ are equal to the forward chamber wall area of area $A_{c}\left(A_{i}=A_{f}=A_{c}\right)$ which is also the cross sectional Area of the entire enclosed OFFPC chamber (Penn state University, 2017). Therefore applying the correlating values to Eq.68, Eq. 68 takes on a value such that:

$$
\oint \rho_{s m} v(v \bullet n) d A=-v_{s m} A_{c} \rho_{s m}
$$

Substituting, the value of Eq.67 and Eq.69 into Eq.66 gives the momentum conservation equation in the form of a differential equation such that:

$$
\frac{d p_{s m}}{d \Delta t}=m_{s m} \frac{d v}{d \Delta t}-v_{s m} A_{c} P_{s m}
$$

This can equivalently be expressed such that $\left(F_{s m}=m_{s m} d v / d \Delta t\right)$ :

$$
F_{g m}=m_{s m} \frac{d v}{d \Delta t}-v_{s m} A_{c} p_{s m}
$$

Algebraically rearranging Eq.71 above and after, solving for volume element $d v$ gives: 


$$
m_{s m} \frac{d v}{d \Delta t}=F_{s m}+v_{s m} A_{c} \rho_{s m} \quad \rightarrow \quad \int d v=\int \frac{d \Delta t}{m_{s m}}\left[F_{s m}+v_{s m} A_{c} \rho_{s m}\right]
$$

Thus after evaluating both integrals in Eq.72, velocity value $v$ assumes a value of:

$$
v=\frac{\Delta t}{m_{s m}}\left[F_{s m}+v_{s m} A_{c} P_{s m}\right]
$$

The conservation of momentum when applied to any system (either a fluid or solid object) states that with no external force acting on it, the variations of momentum in the system will equal the sum of momentum values initially introduced to the system, and therefore equal a constant. Thus, the velocity value of Eq.73 above is a solution to the conservation of momentum equation in a controlled volume system (Eq.64) (Young \& Freedman, 2004) (The physics classroom, 2017). Conclusively, this shows that the OFFPC obeys the law of conservation of momentum in a controlled volume system.

An additional consideration is that the OFFPC does not only compass potential propulsion designs that feature three nozzles as depicted in this paper. OFFPC designs can incorporate multiple nozzles that are perpendicular to the $\mathrm{x}$-axis nozzle i.e. various designs can feature more than two y-axis nozzles and thus more than two y-axis fluid flow streams.

\section{The Generation of Adhesive Force $F_{\text {ema }}$ via the Forward Chamber Wall}

Section 3 introduces the formulation of the equation that describe the adhesive electromagnetic force generated by the forward chamber wall introduced in the previous section. The condition to which the forward chamber wall can produce a current sufficient to generate a negatively charged electric field and therefore an electric force $F_{\text {ema }}$ strong enough to trap the positively charged ionized propellant (satisfying the inequality of Eq.54-55) will be derived. The equation for electric force $F_{e m a}$ is given such that (Young \& Freedman, 2004):

$$
F_{e m a}=e E_{x}
$$

Where $E_{x}$ is the value of the electric field generated by the forward chamber wall and $e$ is the negative charge of the electron (Young \& Freedman,2004). Electric field $E_{x}$ can be expressed such that (Young \& Freedman, 2004):

$$
E_{x}=\frac{F_{\text {gman }}}{\theta}
$$

Electric field $E_{x}$ generated by the forward chamber wall can be expressed as a differential equation, where voltage $V$ is differentiated in respect to spatial length $x$ as shown below (Young \& Freedman, 2004).

$$
E_{x}=-\frac{\partial V}{\partial x}
$$

Thus, Eq.75 is substituted into Eq.76 and gives the equivalence:

$$
\frac{F_{\text {amm }}}{\omega}=-\frac{\partial V}{\partial x}
$$

This can be rearranged such that:

$$
\partial V=-\frac{F_{\mathrm{gma}}}{\bullet} \partial x
$$

Therefore integrating both sides of Eq.78 in respect to voltage $V$ and length $x$ gives (Young \& Freedman, 2004): 


$$
\int_{V_{i}=0}^{V_{f}=V_{\text {lma }}} \partial V=\int_{x_{i}=0}^{x_{f}=\zeta}-\frac{F_{a m a}}{\theta} \partial x
$$

Where the limits of integration for the integrals of Eq.79 are from zero to forward chamber wall voltage $V_{\text {ema }}$ and from zero to length $\zeta$, one obtains the equation shown below (Young \& Freedman, 2004).

$$
V_{e m a}=-\zeta \frac{F_{p m a}}{a}
$$

Where $I$ is the value of current and $R$ is the corresponding resistance within the forward chamber wall; voltage $V_{\text {ema }}$ is expressed such that (Young \& Freedman, 2004):

$$
V_{\text {ema }}=I R
$$

Substituting the value of Eq. 80 into Eq. 81 gives (Young \& Freedman, 2004):

$$
I R=-\zeta \frac{F_{g m a}}{e}
$$

Solving Eq. 82 for electric force $F_{\text {ema }}$ gives Eq. 83 below which is the equation attractive electric force generated by the forward chamber wall.

$$
F_{\text {ema }}=-\frac{e I R}{\zeta} \equiv-\frac{e V_{\text {gma }}}{\zeta}
$$

Lastly, substituting the value of electric force $F_{e m a}$ into the inequality of Eq. 54-55 gives:

$$
\left|F_{e m a}\right|>\left|F_{g m}\right| \quad \rightarrow \quad\left|-\frac{\varepsilon V_{g m a}}{\zeta}\right|>>\left|\frac{A_{c} \rho_{x}\left(V_{x x}\right)^{2}}{2}+A_{c}\left(\frac{\rho_{1 y}\left(\mathbb{V}_{1 y}\right)^{2}}{2}+\frac{\rho_{2 y}\left(\mathbb{V}_{2 y}\right)^{2}}{2}\right)\right|
$$

The Inequality of Eq.84 above is the required condition and value of voltage or electric current that the forward chamber wall of the enclosed chamber would have to generate in order to trap the positively charged ionized propellant. This permits the adhesion of the propellant mass to the vehicle mass which allows the required inelastic collision resulting in propulsion using the orthogonal fluid force vector propulsion concept.

\section{The Repulsive Force of the Side Chamber Walls}

At this juncture, we introduce the formulations of the equations describing the repulsive electric force generated by the side chamber wall which confines or suspends the ionized propellant to the center of the enclosed chamber. Area $A^{y}$ is the symmetric surface area of both upper and lower plates of capacitors built into the chamber side walls which are all separated from one another by a distance $d_{g}$ as shown in figure 3. Insulated wires depicted on the lower plate (or chamber side wall) in figure 3 are conducting a current $I_{B}$ routed into the enclosed chamber and directs the current into the side chamber wall plate in a perpendicular orientation (at the connection points) as depicted in figure 3. 


\section{Upper conducting plate surface}

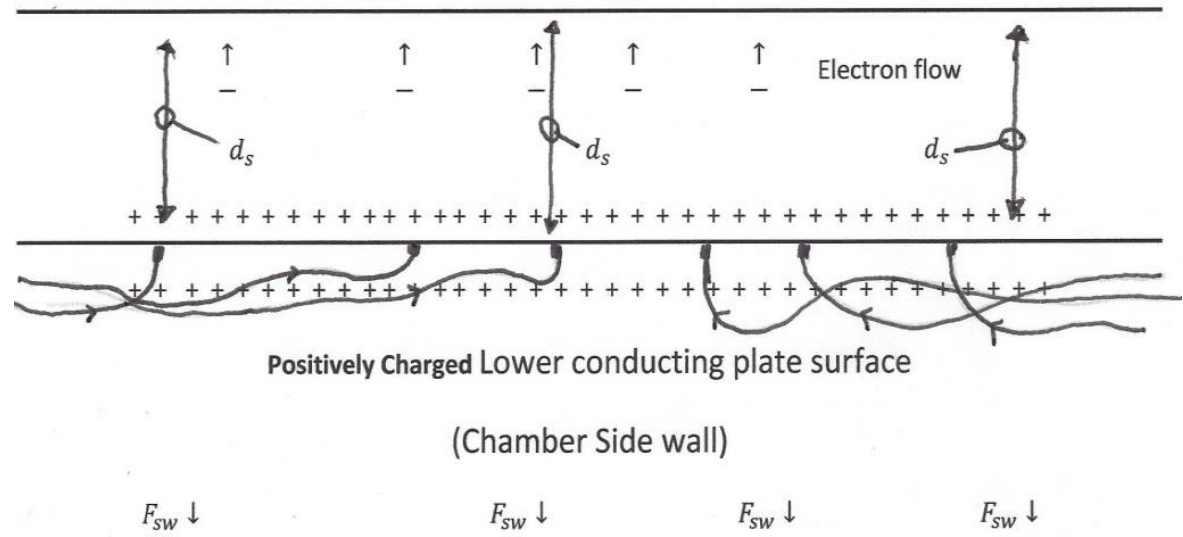

Figure 3. The repulsive force of the chamber side wall

A voltage of $V_{B}$ is conducted between the two plates. Where $C$ is the capacitance and $Q$ is the charge of the chamber side wall plates, voltage $V_{B}$ can be expressed as Eq.85 below (Young \& Freedman, 2004).

$$
V_{B}=\frac{Q}{C}
$$

Expressing voltage $V_{B}$ in terms of current $I_{B}$ and resistance $R$ gives Eq. 86(Young \& Freedman, 2004) .

$$
V_{B}=I_{B} R
$$

Capacitance $C$ can be expressed in terms of chamber wall area $A^{v}$, distance $d_{g}$ between the two plates, and the permittivity of free space $\varepsilon_{0}$ such that(Young \& Freedman, 2004) :

$$
C=\frac{E_{0} A^{f}}{d_{x}}
$$

As previously mentioned, the insulated wires carry current $I_{B}$ up into the bottom plate (or the chamber side wall) and the upper plate hence conducting voltage $V_{B}$ and thus a flow of electrons from the bottom plate to the top plate (Young $\&$ Freedman, 2004). Resultantly, this causes a positive charge $+Q$ on the chamber side wall or bottom plate as electrons transit to the upper plate (Young \& Freedman, 2004). This acquired positive charge on the lower plate which is the chamber side wall allows the repulsion of the positively charged ionized propellant from the forward chamber wall, thus Eq.85 can algebraically manipulated and expressed in terms of positive charge $+Q$ as follows (Young \& Freedman, 2004):

$$
+Q=C V_{B}
$$

Charge $+Q$ can be expressed in terms of the permittivity of free space $\varepsilon_{0}$, the area $A^{s}$ of both plates, and electric field $E_{b}$ as shown below(Young \& Freedman, 2004).

$$
+Q=\epsilon_{0} A^{0} E_{b}
$$

Force $F_{\mathscr{S W}}$ (sw denotes "side wall") is the repulsive force of the side wall chambers (Young \& Freedman, 2004). Where $E_{b}$ is the electric field associated with the chamber wall plate, electric field $E_{b}$ can be expressed in terms of electron charge $e$ and electric force $F_{G W}$ associated with the chamber side wall (i.e. the lower plate) as shown below(Young \& Freedman, 2004) .

$$
E_{b}=\frac{E_{S V}}{e}
$$


Substituting Eq.90 into Eq.89 gives charge $+Q$ such that (Young \& Freedman, 2004):

$$
+Q=\frac{E_{G} A^{s} F_{S Q}}{e}
$$

Substituting Eq.86 and Eq.87 into Eq.88 alternatively gives charge $+Q$ such that(Young \& Freedman, 2004) :

$$
+Q=\frac{\epsilon_{0} A^{r}}{d_{s}} \cdot I_{B} R
$$

Setting equation Eq.91 equal to Eq.92 gives:

$$
\frac{E_{G} A^{s} F_{S V}}{\theta}=\frac{E_{0} A^{s}}{\mathbb{d}_{S}} \cdot I_{B} R
$$

Thus solving Eq.93 for electric force $F_{S W}$ generated by the side chamber wall plate gives the result in terms of current $I_{E}$, resistance $R$, electron charge $e$, and the distance between the chamber wall plate and the lower plate $d_{g}$ gives:

$$
F_{G W}=\frac{I_{B} R E}{d_{s}}
$$

Thus one obtains the equation of the repulsive positively charged force $F_{S W}$ generated by the side walls of the enclosed chamber which confines the positively charged ionized propellant to the center of the enclosed chamber preventing the ionize propellant from coming into contact with the side chamber walls resultantly prevents exertion the $\mathrm{x}$-axis between the ends of the chamber.

\section{Energy Regeneration within the OFFPC}

A portion or all of the power used in various potential designs incorporating the orthogonal fluid flow propulsion concept (OFFPC) will be produced by electricity. The advantage to the OFFPC is the possibility of regenerating a portion of the electricity lost thus contributing to the longevity of the power sources. Regeneration in the OFFPC is analogous to a car battery and an alternator. The average automobile battery will run for an approximate total of six hours without an alternator, however, with an alternator, the average car battery can last for up to six years. The property of electrical regeneration in the OFFPC allows longevity to possible designs that are battery powered or have reserve systems. Section 3 shows the mathematical process by which this is achieved. Figure 4 below, shows the ionized propellant that is trapped onto the forward chamber wall by electrical force $\left|F_{\text {ema }}\right|$ which egresses through the recycle valves which lead to the generator turbine. Recall that once trapped, the velocity of the ionized propellant is zero relative to the motion of the entire vehicle. In this explanation, the design incorporates only two recycle valves, this is important to calculations.

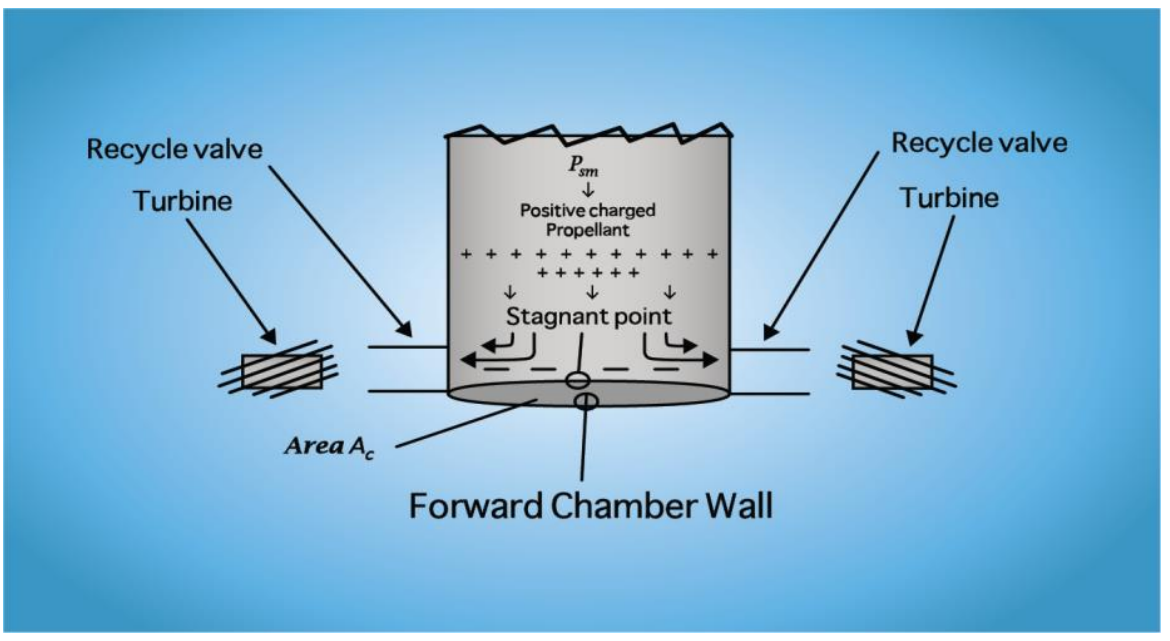

Figure 4. The Recycle valves 
The pressure $P_{\text {ema }}$ is the pressure value exerted on the ionized fluid by the electrical force value $\left|F_{\text {ema }}\right|$ i.e. pressure value $P_{e m a}$ is the force of $\left|F_{e m a}\right|$ over area $A_{c}$ and thus over the forward chamber wall. Pressure value $P_{e m a}$ is expressed as Eq.95 below (Hyper physics, 2017) (Young \& Freedman, 2004).

$$
P_{\text {ema }}=\frac{\left|F_{\text {sma }}\right|}{A_{\varepsilon}}
$$

This implies that (Young \& Freedman, 2004):

$$
\left|F_{\text {ema }}\right|=P_{\text {ema }} A_{c}
$$

The density of the suspended ionized propellant $\rho_{s m}$ within the enclosed chamber is the fluid mass of $m_{x}+m_{1 y}+m_{2 y}$ per volume $V_{c}$ of the entire enclosed chamber $\left(\left(\rho_{s m}=\left(m_{x}+m_{1 y}+m_{2 y}\right) / V_{c}\right)\right.$. A stagnate point in the center of area $A_{c}$ of the forward chamber wall divides the fluids in half for the two recycle valves, thus the value of masses of $m_{x}+m_{1 y}+m_{2 y}$ is divided by two $\left(\left(m_{x}+m_{1 y}+m_{2 y}\right) / 2\right)$. Therefore, the density of the fluids flowing into both recycle valves is half the value of density $\rho_{s m}\left(\rho_{s m} / 2\right)$. Therefore, density $\rho^{s}$ is half of density $\rho_{s m}$ $\left(\rho^{s}=\left(\rho_{s m} / 2\right)\right)$ and is the density of the fluid egressing through each recycle valve. Density $\rho^{s}$ can be expressed as Eq. 97 below.

$$
\rho^{s}=\frac{\rho_{s m}}{2}=\frac{m_{x}+m_{1 y}+m_{2 y}}{2 V_{c}}
$$

The fluid velocity becomes zero relative to the motion of the entire vehicle upon impact with the forward chamber wall as the fluid transfers its kinetic energy (and momentum) to the entire vehicle. Thus, the electric force of $\left|F_{\text {ema }}\right|$ generated by the forward chamber wall essentially squeezes the fluid out of the chamber via the two recycle valves located alongside the forward chamber wall. Electric force $\left|F_{\text {ema }}\right|$ exerts on the fluids on the forward chamber wall producing pressure $P_{\text {ema }}$ of Eq.95, as the propellant velocity is zero relative to the motion of the entire vehicle. As shown in figure 4 , the fluid exiting the chamber exerts an aerodynamic force $F_{B}$ on the turbines within the recycle valves which is equal to the force exerted on the fluid by electromagnetic force $\left|F_{e m a}\right|$ (i.e. $P_{e m a} A_{\varepsilon}$ ) (Young $\&$ Freedman, 2004) .

$$
F_{B}=\frac{N_{B} A_{B} V_{B}^{2} \rho^{\prime} \sin \theta_{B}}{2} \equiv P_{e m a} A_{C}
$$

Where $N_{B}$ denotes the discrete number of turbine blades, area $A_{B}$ denotes the area of each turbine blade, velocity $v_{B}$ is the tangential velocity of all of the turbine, angle $\theta_{B}$ is the angle of incident of each turbine blade and $\rho^{\Delta}$ (of Eq.97) is fluid density of each valve. The aerodynamic force of the fluid (or ionized gas) flowing through each recycle valve exerting on the turbines is equal to electric force $\left|F_{\text {ema }}\right|$. Hence, aerodynamic force $F_{B}$ is set equal to force $\left|F_{\text {ema }}\right|$ (of Eq. 83) as shown in Eq. 99.

$$
\left|F_{\text {ema }}\right|=F_{B}
$$

Eq.99 can alternatively be expressed in terms of Eq. 83 such that:

$$
\left|-\frac{\theta V_{g m a}}{\zeta}\right|=\frac{N_{B} A_{B} V_{B}^{2} \rho^{r} \sin \theta_{B}}{2}
$$

Where,

$$
\left|F_{e m a}\right|=\left|-\frac{e V_{a m a}}{\zeta}\right|=\frac{e V_{a m a}}{\zeta}
$$

Thus solving for tangential velocity $v_{B}$ (shown in figure 5) gives Eq.102 as shown below. 


$$
v_{B}=\sqrt{\frac{2\left|F_{\text {gma }}\right|}{N_{B} A_{B} \rho^{f} \sin \theta_{B}}}
$$

The friction of the spinning turbine is negligible for the purposes of this derivation. Figure 4 below shows one of the turbines rotating a copper wire within a perpendicular magnetic field $B$ at a tangential velocity of $v_{B}$ resulting from the egressing fluids.

\section{OFFPC Regenerator turbine}

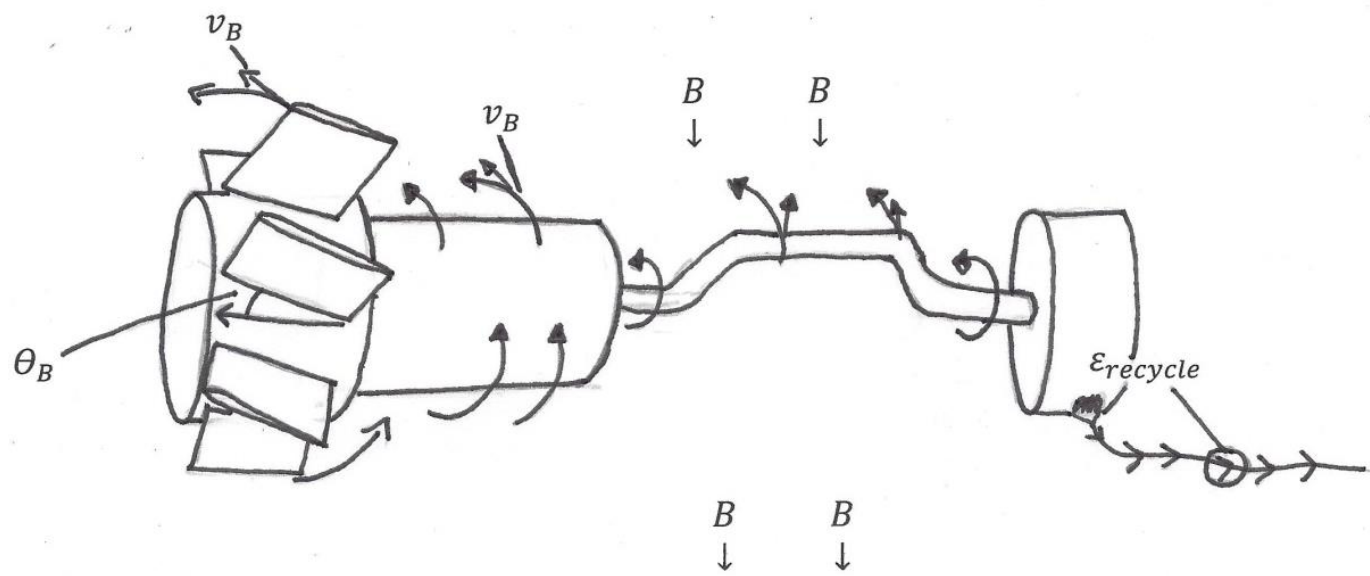

Figure 5. The regeneration turbine

Consider the relationship of tangential velocity $v$ to angular velocity $\omega$ and radius $r(v=r \omega)$ traveled by the rotating wire (Young \& Freedman, 2004). Angular velocity $\omega$ can be expressed such that (Young \& Freedman, 2004):

$$
\omega=\frac{v}{r}
$$

Where radius $r$ is the radial distance from to the center of rotation of the rotating copper wire, angular velocity $\omega_{B}$ corresponds to tangential velocity $v_{B}\left(v=v_{B}\right)$ produced by the egressing ionized propellant rotating the turbine given by Eq. 104 below (Young \& Freedman,2004).

$$
\omega_{B}=\frac{W_{B}}{r}
$$

Substituting Eq.102 into Eq.104 gives angular velocity $\omega_{B}$ such that (Young \& Freedman, 2004):

$$
\omega_{B}=\frac{1}{Y} \sqrt{\frac{2] F_{g m a} \mid}{N_{B} A_{B} \rho^{I} \sin \theta_{B}}}
$$

Consider the electric and magnetic flux $\phi_{B}$ of Faraday's law incorporating angular velocity $\omega_{B}$ of Eq.106 below (Young \& Freedman, 2004).

$$
\phi_{B}=B A \cos \left(\omega_{B} t\right)
$$

Where $B$ is the magnetic field encompassing the rotating the copper wire and $A$ is the spatial area encompassed by the magnetic field, the time derivative of flux $\phi_{B}$ gives the induced EMF $\varepsilon$ (Young \& Freedman, 2004).

$$
\varepsilon_{\text {recycle }}=\frac{d \phi_{B}}{d t}=-\omega_{B} B A \sin \left(\omega_{B} t\right)
$$


Hence, Eq.108 below gives the total voltage $\varepsilon_{\text {recycle }}$ regenerated by a single valve of the OFFPC (Young \& Freedman, 2004).

$$
\varepsilon_{\text {recycle }}=I_{\text {recycled }} R
$$

Where $I_{\text {recycled }}$ and $R$ are the electric current and resistance of the rotating copper wire (Young \& Freedman, 2004),

$$
\varepsilon_{\text {yecycle }}=-\omega_{B} B A \sin \left(\omega_{B} t\right)
$$

Eq.107 can be alternatively expressed such that (Young \& Freedman, 2004):

$$
l_{\text {recycled }} R=-\omega_{B} B A \sin \left(\omega_{B} t\right)
$$

Current $l_{\text {recycled }}$ is the portion of the electrical current sent to replenish the battery or power source allowing the longevity of the power source (or reserve system). Substituting Eq.106 into Eq.110; Eq.110 can alternatively be expressed such that (Young \& Freedman, 2004):

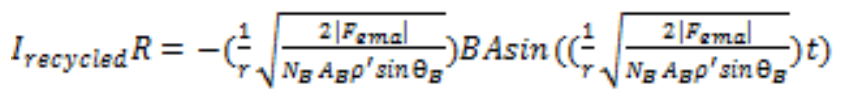

Thus Eq.111 above gives a mathematical expression of the potential electric power regenerated by the OFFPC allowing the longevity of electrical power sources in various designs.

\section{Energy and Power Loss to the OFFPC System}

Similarly to the EM Drive, the OFFPC will inherently be interpreted as a perpetual motion device. This section shows the power (and thus energy) loss experienced by the system as it operates. Similarly to the EM Drive, the OFFPC is an electrically driven system and does not lose mass due to the fact that the ionized propellant is recyclable. Therefore, the total amount of electric potential (or voltage) used to accelerate the propellant will be denoted $V_{\text {prop }}$. Thus electric

potential $V_{\text {prop }}$ can be expressed such that (Young \& Freedman, 2004):

$$
V_{\text {prop }}=I R
$$

Where $I$ is the electric current and $R$ is the resistance of the conducting medium, the corresponding value of electric potential energy $U_{\text {prop }}$ is expressed such that (Young \& Freedman, 2004):

$$
U_{\text {prop }}=q V_{\text {prop }}
$$

Where $q$ is the corresponding value of charge (Young \& Freedman, 2004), electric potential energy $U_{\text {prop }}$ is the total amount of energy required to accelerate the ionized propellant out of all three fluid nozzles. Potential energy $U_{\text {prop }}$ is then converted to kinetic energy $K_{s m} \quad\left(K_{s m}=V_{c} P_{s m}\right.$ of Eq. 28) which is the energy of the suspended mass of all three propellant fluid flows. Therefore, electric potential energy $U_{\text {prop }}$ is equal to the energy of the suspended propellant mass of kinetic energy $K_{g m}$ of Eq.27-28 as shown below (Young \& Freedman, 2004). 


$$
U_{\text {prop }}=K_{s m}
$$

Or alternatively,

$$
q V_{\text {prop }}=\frac{V_{c} \rho_{x}\left(V_{x}\right)^{2}}{2}+V_{c}\left(\frac{\rho_{1 y}\left(V_{1 y}\right)^{2}}{2}+\frac{\rho_{2 y}\left(V_{2 y}\right)^{2}}{2}\right)
$$

Where $V_{c}$ denotes the volume of the enclosed chamber, consider the vehicle velocity $v_{b}$ of Eq.69 gained by the vehicle per time value $\Delta t$ shown below.

$$
v_{v}=\frac{\Delta t}{\left(m_{v}+m_{x}+m_{1 y}+m_{2 y}\right)}\left[\left(A_{c}\left(\frac{\rho_{1 y}\left(v_{1 y}\right)^{2}}{2}+\frac{\rho_{2 y}\left(v_{2 y}\right)^{2}}{2}\right)\right)\right]
$$

The kinetic energy $k_{w}$ acquired by the vehicle as a result of propulsion generated by the OFFPC which is a function of vehicle velocity $v_{b}$ is expressed as (Young \&Freedman, 2004):

$$
k_{v}=\frac{\left(w_{v}\right)^{2}}{2}\left(m_{v}+m_{x}+m_{1 y}+m_{2 y}\right)
$$

Substituting Eq.116 into Eq.117 allows Eq.117 above to alternatively be expressed such that:

$$
k_{v}=\frac{(\Delta t)^{2}}{2\left(m_{v}+m_{x}+m_{1 y}+m_{2 y}\right)}\left[\left(A_{c}\left(\frac{\rho_{1 y}\left(v_{1 y}\right)^{2}}{2}+\frac{\rho_{2 y}\left(v_{2 y}\right)^{2}}{2}\right)\right)\right]^{2}
$$

The value of kinetic energy $k_{v}$ of Eq.118 above generated by the OFFPC is merely a portion of the total electrical potential energy value $U_{\text {prop }}$ required to accelerate the ionized propellant. Thus, the value of vehicle kinetic energy $k_{\mathrm{b}}$ inherited by the entire vehicle is less than the total amount of electrical potential energy $U_{\text {prop }}\left(U_{\text {prop }}>k_{w}\right)$ required to accelerate the ionized propellant per time span $\Delta t$. Thus, out of all of the energy required to accelerate the ionized propellant, only a smaller portion of energy is actually converted to thrust. The total energy generated by the vehicle's power source which operates the entire OFFPC propulsion system is denoted $E_{\text {output }}$ which is the energy value per time $\Delta t$ i.e. power value $P_{\text {prop }}\left(P_{\text {prop }}=E_{\text {output }} / \Delta t\right.$ ) (Young \& Freedman, 2004). Energy $E_{\text {output }}$ is the sum of electric energy values required to operate the propulsion system, energy $E_{\text {output }}$ can be expressed such that:

$$
E_{\text {output }}=U_{\text {prop }}+q V_{\text {ema }}+\Sigma q V_{B}
$$

Where $q$ is the corresponding charge value, the electric potential energy $q V_{e m a}$ (for electric potential $V_{e m a}$ of adhesive force $F_{e m a}$ ) required to trap the ionized propellant on the forward chamber wall, and the sum of electric potential energy values $\Sigma q V_{B}$ (for electric potential sum $\Sigma V_{B}$ ) required to generate the repulsive forces of the all of the chamber side walls (of force value $F_{G V}$ ) (Young \& Freedman,2004). As expressed in the previous section, the turbines within the recycle valves to which the propellant egresses permits regeneration of voltage $\varepsilon_{\text {recycie }}$ which is returned to the power source or other function (Young \& Freedman, 2004). Thus, the electric potential energy corresponding to voltage $\varepsilon_{\text {yecycle }}$ (of Eq.107-109) is expressed as $q \varepsilon_{\text {recycle }}$ for charge $q$ (Young \& Freedman, 2004). The electric potential $q \varepsilon_{\text {recycle }}$ pertains to one recycle valve, where the design presented in this paper incorporates 2 recycle valves of equal magnitude, therefore the total electric energy generated by both recycle values is given by $2 q \varepsilon_{\text {recycle }}$. Thus, the portion of energy $E_{\text {output }}$ used for motion and the energy returned to the system via regeneration is the sum of vehicle kinetic energy $k_{w}$ (of Eq.118) and electric potential energy $2 q \varepsilon_{\text {recyele }}$ which is expressed below.

$$
k_{w}+\left|2 q \varepsilon_{\text {recycle }}\right|
$$

Eq.120 above shows the absolute value of electric potential $2 q \varepsilon_{\text {recycle }}$ above (e.g. $\left|2 q \varepsilon_{\text {recycie }}\right|$ ). The sum of energies of Eq. 120 is a portion of energy $E_{\text {output }}$, hence the sum of energies (i.e. $\left.k_{v}+\left|2 q \varepsilon_{\text {recyele }}\right|\right)$ must be less than the total energy output or energy $E_{\text {output }}$ (Eq.119) as conveyed by the inequality below. 


$$
E_{\text {output }}>k_{v}+\left|2 q \varepsilon_{\text {recyele }}\right|
$$

The portions of energy that has not been used for motion or regeneration is denoted $E_{\text {excess }}$. Energy $E_{\text {excess }}$ is the difference of energy $E_{\text {output }}$ and the sum of energies $k_{v}+q \varepsilon_{\text {recycle }}$ as shown below.

$$
E_{\text {excess }}=E_{\text {output }}-\left[k_{v}+\left|2 q \varepsilon_{\text {recyele }}\right|\right]
$$

The pertinent question is what becomes of the excess energy $E_{\text {excess }}$. The answer is that the excess energy $E_{\text {excess }}$ is converted into thermal energy within the propellant that is transferred to the structure of the enclosed chamber i.e. the energy is simply converted to heat. The transfer of energy from the propellant to the structure of the chamber occurs when the propellant contacts the forward chamber wall. Hence, we begin with the molecular energy of the ionized propellant. The kinetic energy $k_{\text {ty }}$ of all of the molecules of the ionized propellant has a value such that (Hyper Physics, 2017) (Young \& Freedman, 2004):

$$
k_{t y}=\frac{N m_{r}\left(v_{r m s}\right)^{2}}{2}=\frac{a N T k}{2}
$$

Where $v_{r m s}$ is the root-mean-square speed of molecules composing the propellant, mass $m_{y}$ is the molecular mass of each molecule composing the ionized propellant and $k$ is the Boltzmann constant(Young \& Freedman ,2004). In Eq.123, $N$ is the number of molecules within the propellant. Thus molecule number $N$ has a value such that (Young $\&$ Freedman, 2004):

$$
N=\left(\frac{m_{\text {tot }}}{M_{\text {mol }}}\right) \times N_{a}
$$

Where $N$ is the number of molecules within the propellant, $m_{\text {tot }}$ denotes the mass or total mass of the propellant, $M_{\text {mol }}$ denotes the molar mass of the propellant, and $N_{\alpha}$ denotes Avogadro's constant (Young \& Freedman,2004). In expressing kinetic temperature $T$ in terms of molecular kinetic energy $k_{\text {ty }}$ gives a value such that (Hyper Physics, 2017) (Young \& Freedman, 2004):

$$
T=\frac{21}{a k}\left[k_{t y}\right] \equiv \frac{2}{a R n}\left[k_{t y}\right]
$$

This can alternatively be expressed such that (Young \& Freedman, 2004):

$$
T=\frac{21}{a k}\left[\frac{\mathrm{Nm} m_{r}\left(\mathrm{~V}_{r m s}\right)^{2}}{2}\right] \equiv \frac{2}{\mathrm{aRn}}\left[\frac{\mathrm{Nm} r\left(\mathrm{~W}_{r m s}\right)^{2}}{2}\right]
$$

Where $R$ is the gas constant and $n$ denotes the number of moles, the value of molecular velocity $v_{r m s}$ can be expressed such that (Young \& Freedman, 2004):

$$
v_{r m s}=\sqrt{\frac{a h T}{m_{r}}}=\sqrt{\frac{a n n T}{N}}
$$

Molecular velocity $v_{\text {rms }}$ is also equal to the square root of the square velocity $\left(v_{a v}\right)^{2} \quad\left(v_{r m s}=\sqrt{\left(v_{a v}\right)^{2}}\right)$, where $\left(v_{a v}\right)^{2}$ is the average molecular velocity of the propellant and has a value of (Young \& Freedman, 2004):

$$
\left(v_{a v}\right)^{2}=\left(v_{a v x}\right)^{2}+\left(v_{a v y}\right)^{2}+\left(v_{a v z}\right)^{2}
$$

Due to the fact that all of the (repulsive or attractive) forces generated within the enclosed chamber enact on the ionized propellant, the energy of $E_{\text {excess }}$ (which is a result of all of the enacting forces) is set equal to the molecular kinetic energy pertaining to the ionized propellant $\left(E_{E x c e s s}=k_{t y}\right)$, where $N$ is the number of molecules within the propellant as shown below (Young \& Freedman, 2004). 


$$
\frac{N m_{r}\left(\mathbb{V}_{\text {rmss }}\right)^{2}}{2}=E_{\text {excess }} \equiv E_{\text {output }}-\left[k_{v}+\left|2 q \varepsilon_{\text {recyele }}\right|\right]
$$

Therefore, the kinetic temperature equation which is the temperature $T$ inherited by the ionized propellant can be alternatively expressed in terms of the value of excess energy $E_{\text {expended }}$ such that (Young \& Freedman, 2004):

$$
T=\frac{2}{a k}\left[E_{\text {output }}-\left[k_{v}+2 q \varepsilon_{\text {recycie }}\right]\right]=\frac{2}{a R n}\left[E_{\text {output }}-\left[k_{v}+\left|2 q \varepsilon_{\text {recycie }}\right|\right]\right]
$$

Thus the kinetic temperature $T$ is the temperature acquired by the ionized propellant which is transferred to the vehicle structure. Resultantly, a portion of the energy will leave the system in the form of thermal photons i.e. thermal radiation contingent on the ambient temperature (Hyper Physics, 2017) (Young \& Freedman, 2004). The equation for power $\left(P_{\text {radiation }}\right)$ corresponding to the radiant energy emitted from the vehicle structure as the heat energy is transferred from the ionized propellant described by the Stefan-Boltzman law equation displayed below (Hyper Physics, 2017) (Young \& Freedman, 2004).

$$
P_{\text {radiation }}=A_{p} \theta \sigma\left(T^{4}-T_{K}^{4}\right)
$$

Where $e$ is the emissivity, $A_{p}$ is the area through which the radiation propagates, $\sigma$ is Stefan's constant, and $T_{k}$ denotes the ambient temperature which is the temperature of the structure of the enclosed chamber and thus the surrounding vehicle (Hyper Physics, 2017) (Young \& Freedman, 2004). Thus, the thermal radiation leaving the entire vehicle as it pertains to energy $E_{\text {excess }}$ can be expressed such that:

$$
P_{\text {radiation }}=A_{p} e \sigma\left(\left[\frac{2}{a k}\left[E_{\text {output }}-\left[k_{v}+\left|2 q \varepsilon_{\text {recycle }}\right|\right]\right]\right]^{4}-T_{K}^{4}\right)
$$

However, here we exclusively focus on the heat or radiant energy transferred to the structure of the enclosed chamber denoted $H_{\text {propellant }}$ which has a value such that (Hyper Physics, 2017) (Young \& Freedman, 2004):

$$
H_{\text {propellant }}=A_{p} e \sigma T^{4}
$$

Substituting the value of temperature $T$ of Eq.130 into Eq.133 gives the heat value transferred to the structure of the enclosed chamber such that (Hyper Physics, 2017) (Young \& Freedman, 2004):

$$
H_{\text {propellant }}=A_{p} \theta \sigma\left[\frac{2}{a k}\left[E_{\text {output }}-\left[k_{v}+\left|2 q \varepsilon_{\text {recycie }}\right|\right]\right]\right]^{4}
$$

In conclusion, a portion of the electrical energy $E_{\text {output }}$ is used to produce thrust (which is kinetic energy $k_{v}$ ) in the OFFPC and the other portion is partly returned while the rest is converted to heat and thus thermal energy which is partially emitted as radiation. Moreover, this shows that energy is lost by an OFFPC system as with conventional systems and must expend energy to continue to produce thrust.

\section{Conclusion: Quantitative Results and a Hypothetical Demonstration}

The most efficient way to show the plausibility of the OFFPC is to apply values of associated quantities to the equations and expressions of the OFFPC, thus describing a hypothetical motor. Therefore, the potential operation of the OFFPC will now be demonstrated via a hypothetical motor. Table 1 below contains the values to be used in the mathematical demonstration. 
Table 1. The values to be used in the mathematical demonstration

\begin{tabular}{|c|c|}
\hline Variable- & Quantity type $\cdots$ \\
\hline$V_{c} \cdots \cdots \cdots$ & Enclosed chamber Volume $\cdots$ \\
\hline$m_{x} \cdots \cdots \cdots \cdots$ & $\begin{array}{l}\text { Propellant mass out of the } \mathrm{x} \text {-axis nozzle } \\
\text { (note: these are mass values per } \mathrm{sec} \text { ) }\end{array}$ \\
\hline$m_{y 1}=m_{y 2}$ & $\begin{array}{l}\text { Propellant mass out of the } \mathrm{y} \text {-axis nozzles } \\
\text { (note: these are mass values per } \mathrm{sec} \text { ) }\end{array}$ \\
\hline$m_{v} \cdots \cdots$ & Mass of the entire vehicle \\
\hline$v_{x} \cdots \cdots \cdots$ & Propellant velocity out of the $\mathrm{x}$-axis nozzle \\
\hline$v_{y 1}=v_{y 2}$ & Propellant velocity out of each $1 \times 10^{5} \mathrm{~m} / \mathrm{s}$ \\
\hline$A_{c} \cdots \cdots \cdots$ & Area of the forward chamber wall \\
\hline$\zeta \cdots \cdots \cdots \cdots \cdots$ & Length corresponding to voltage value $V_{\text {ema }} \ldots$ \\
\hline$e$ & Charge of an electron $\cdots$ \\
\hline$\Delta t=t \cdots \cdots$ & Time span or time interva1 \\
\hline
\end{tabular}

Applying the values of Table 1, the fluid density $\rho_{x}$ corresponding to the nozzle on the x-axis or direction of travel takes on a value such that:

$$
\rho_{x}=\frac{m_{x}}{V_{c}}=\frac{a \times 10^{-15}}{V_{c}}=4.24 \times 10^{-14} \mathrm{~kg} / \mathrm{m}^{\mathrm{a}}
$$

Due to the fact that $\rho_{1 y}=\rho_{2 y}$, the densities $\rho_{1 y}$ and $\rho_{2 y}$ corresponding to the perpendicular opposing nozzles on the $y$-axis are given such that:

$$
\begin{aligned}
& \rho_{1 y}=\frac{m_{1 y}}{v_{c}}=\frac{1 \times 10^{-15_{\mathrm{kg}}}}{v_{c}}=1.41 \times 10^{-14} \mathrm{~kg} / \mathrm{m}^{\mathrm{a}} \\
& \rho_{2 y}=\frac{m_{2 y}}{v_{c}}=\frac{1 \times 10^{-15} \mathrm{~kg}}{v_{c}}=1.41 \times 10^{-14} \mathrm{~kg} / \mathrm{m}^{a}
\end{aligned}
$$

Hence, the dynamic pressure value $P_{x}$ corresponding to the flow of ionized propellant injected into the enclosed chamber on the $\mathrm{x}$-axis has a value such that:

$$
P_{x}=\frac{\rho_{x}\left(\mathbb{W}_{x)^{2}}\right)^{2}}{2}=3.39 \times 10^{-9} \mathrm{~N} / \mathrm{m}^{2}
$$

Therefore, the dynamic pressure values $P_{1 y}$ and $P_{2 y}$ corresponding to the orthogonal flow of ionized propellant on the $y$-axis are given such that;

$$
P_{1 y}=\frac{\rho_{1 y}\left(\mathrm{v}_{1 y}\right)^{2}}{2}=7.05 \times 10^{-5} \mathrm{~N} / \mathrm{m}^{2} \quad P_{2 y}=\frac{\rho_{2 y\left(v_{2 y}\right)^{2}}}{2}=7.05 \times 10^{-5} \mathrm{~N} / \mathrm{m}^{2}
$$

Resultantly, the dynamic pressure of all three fluid streams as they meet in the center of the enclosed chamber are given by pressure $P_{s m}$ of Eq.31 expressed below.

$$
P_{s m}=\frac{\rho_{x}\left(\mathbb{V}_{x}\right)^{2}}{2}+\left(\frac{\rho_{1 y}\left(\mathbb{D}_{1 y}\right)^{2}}{2}+\frac{\rho_{2 y}\left(\mathrm{D}_{2 y}\right)^{2}}{2}\right)
$$


Applying the values of Eq.138 and 139 to Eq.140 above gives a value of suspended mass dynamic pressure $P_{\mathscr{B}}$ shown below.

$$
P_{\mathrm{gm}}=3.53 \times 10^{-\mathrm{a}} \mathrm{N} / \mathrm{m}^{\mathrm{a}}
$$

As previously asserted, the magnitude of the pressure value on the x-axis $P_{x}$ must be greater than the combined sum of pressure values $P_{1 y}$ and $P_{2 y}$ oriented on the perpendicular axis (or y-axis) as conveyed by the inequality below

$$
P_{x}>P_{1 y}+P_{2 y}
$$

Or Alternatively,

$$
\frac{\rho_{x}\left(V_{x}\right)^{2}}{2}>\frac{\rho_{1 y}\left(v_{1 y}\right)^{2}}{2}+\frac{\rho_{2 y}\left(V_{2 y}\right)^{2}}{2}
$$

The inequalities of Eq.142 and Eq.143 above shows that the more powerful fluid stream of $P_{x}$ on the x-axis diverts the direction of the dynamic pressure of orthogonal streams $P_{2 y}$ and $P_{1 y}$ into the direction of the stream of $P_{x}$ towards the forward chamber wall; i.e. all of the fluid streams travel in the same direction on the $\mathrm{x}$-axis. Thus the values of pressure as they pertain to the hypothetical motor obey the inequality such that:

$$
3.39 \times 10^{-9} \mathrm{~N} / \mathrm{m}^{2}>1.41 \times 10^{-4} \mathrm{~N} / \mathrm{m}^{2}
$$

Recall that the ionized propellant is suspended to the center of the chamber via the electric positively charged force $F_{\text {sIV }}$ generated by the side wall. Hence, as the ionized propellant collides with the forward chamber wall of area $A_{c}$ a force value $F_{s m}$ is produce of a value:

$$
F_{s m}=\frac{A_{c} \rho_{x}\left(V_{x x}\right)^{2}}{2}+A_{c}\left(\frac{\rho_{1 y}\left(\mathrm{~V}_{1 y}\right)^{2}}{2}+\frac{\rho_{2 y}\left(\mathrm{~V}_{2 y}\right)^{2}}{2}\right)=6.44 \times 10^{-5} \mathrm{~N}
$$

As expressed in Eq.47, the total thrust value $F_{z^{\prime}}$ on the x-axis of the enclosed chamber is given such that:

$$
F_{X^{\prime}}=\left(\frac{A_{c} \rho_{x}\left(\mathbb{V}_{x}\right)^{2}}{2}+A_{c}\left(\frac{\rho_{1 y}\left(\mathbb{N}_{1 y}\right)^{2}}{2}+\frac{\rho_{2 y}\left(\mathbb{V}_{2 y}\right)^{2}}{2}\right)\right)-\left(v_{x} \dot{m}_{x}+\frac{A_{x} \rho_{x}\left(V_{x}\right)^{2}}{2}\right)
$$

Hence, due to Newton's third law:

$$
\sum F_{x}=F_{x 0}-F_{x}=0 \quad \rightarrow \quad F_{x 0}=F_{x}
$$

The $\mathrm{x}$-axis terms of pressure are required to cancel out as conveyed below.

$$
\frac{A_{c} \rho_{x}\left(V_{x}\right)^{2}}{2}-\left(v_{x} \dot{m}_{x}+\frac{A_{x} \rho_{x}\left(w_{x}\right)^{2}}{2}\right)=0
$$

In this example, Newton's third law is mathematically satisfied by x-axis nozzle opening area $A_{x}$ as shown below.

$$
A_{x}=A_{e}-\frac{2 n_{x}}{\rho_{x} N_{x}}
$$

Eq.149 above must be true for the condition of:

$$
A_{e}>\frac{2 m_{x}}{\rho_{x} W_{x}}
$$


Substituting the given values into the inequality of Eq.150, we observe that the inequality of Eq.150 is satisfied as shown below.

$$
.0182>3.53 \times 10^{-7}
$$

Where the mass per time rate $m_{x}$ expressed in Eq.146, Eq.148, and Eq.149-150 has a values such that:

$$
\dot{m}_{x}=\frac{m_{x}}{\Delta t}=\frac{a \times 10^{-15} \mathrm{~kg}}{1 s}=3 \times 10^{-15} \mathrm{~kg} / \mathrm{s}
$$

Conclusively, the thrust value $F_{x^{\prime}}$ given on the x-axis has a value such that:

$$
F_{x^{\prime}}=A_{c}\left(\frac{\rho_{1 y}\left(N_{1 y}\right)^{2}}{2}+\frac{\rho_{2 y}\left(v_{2 y}\right)^{2}}{2}\right)=2.57 \times 10^{-6} \mathrm{~N}
$$

Applying the values of table 1 , the vehicle acceleration $a_{v}$ takes on a value of:

$$
a_{v}=\frac{1}{\left(m_{v}+m_{x}+m_{1 y}+m_{2 y}\right)}\left[\left(A_{c}\left(\frac{\rho_{1 y}\left(v_{1 y}\right)^{2}}{2}+\frac{\rho_{2 y}\left(v_{2 y}\right)^{2}}{2}\right)\right)\right]=5.97 \times 10^{-6} \mathrm{~m} / \mathrm{s}^{2}
$$

Thus, in producing an inelastic collision which results from the temporary adhesion of propellant mass to the vehicle mass (e.g. $m_{v}+m_{x}+m_{1 y}+m_{2 y}$ ) required to generate vehicle acceleration $a_{y}$ and thrust value $F_{x^{\prime}}$; the electric force $F_{\text {ema }}$ generated by the forward chamber wall must be much greater than force $F_{s m}$ of the suspended propellant mass exerted on the forward chamber wall as shown below.

$$
\left|F_{e m a}\right| \gg\left|F_{g m}\right| \quad \rightarrow \quad|-e I R| \gg \frac{A_{c} \rho_{x}\left(v_{x}\right)^{2}}{2}+A_{c}\left(\frac{\rho_{1 y}\left(v_{1 y}\right)^{2}}{2}+\frac{\rho_{2 y}\left(v_{2 y}\right)^{2}}{2}\right)
$$

Hence, we observe the absolute value of force $F_{\text {ema }}$ generated by the forward chamber wall given such that:

$$
\left|F_{\text {ema }}\right|=\left|-\frac{e V_{\text {ama }}}{\zeta}\right|=1.93 \times 10^{-4} \mathrm{~N}
$$

Therefore, the values of force $F_{\text {ema }}$ and force $F_{s m}$ satisfy the inequality of Eq.155,

$$
1.93 \times 10^{-4} \mathrm{~N} \quad>\quad 6.44 \times 10^{-5} \mathrm{~N}
$$

The value of voltage required to generate forward chamber wall force $F_{\text {ema }}$ is given by the equation of:

$$
V_{\text {ema }}=I R=-\zeta \frac{F_{\text {ema }}}{e}
$$

Applying the values of table 1, the value of forward chamber wall voltage $V_{e m a}$ is given such that:

$$
V_{\text {ema }}=-9.19 \times 10^{1} \text { TeraVolts }
$$

Or Alternatively,

$$
\left|V_{\text {ema }}\right|=9.19 \times 10^{1} \text { TeraVolts }
$$

Admittedly, this voltage value is exorbitantly high however, it is necessary to capture and temporarily stop the propellant producing an inelastic collision. At this juncture, the movement and management of energy within the 
hypothetical motor can be mathematically demonstrated. Table 2 below contains values pertaining to the processes of power and energy within the OFFPC system.

Table 2. The values pertaining to the processes of power and energy within the OFFPC system

\begin{tabular}{|c|c|}
\hline Variable & Quantity type \\
\hline$k$ & Boltzmann constant $\cdots \cdots .1 .38 \times 10^{-23} \mathrm{~m}^{2} \mathrm{~kg} \mathrm{~S}^{-2} \mathrm{~K}^{-1}$ \\
\hline$r$ & Radius from the recycle turbine core to the turbine blade tip $\quad 3.81 \times 10^{-3} \mathrm{~m}$ \\
\hline$N_{B}$ & Number of turbine blades on the recycle valve turbine \\
\hline$\Theta_{B}$ & Angle of incidents for each turbine blade $30^{\circ}$ \\
\hline$B$ & The value of the magnetic field associated with the generator \\
\hline$A \cdots$ & The cross sectional area encompassed by the magnetic field $B \cdots 5.19 \times 10^{-4} \mathrm{~m}^{2}$ \\
\hline$A_{B}$ & Area of each turbine blade \\
\hline$N_{a}$ & Avogadro's number \\
\hline
\end{tabular}

As previously expressed, a stagnate point in the center of area $A_{c}$ of the forward chamber wall divides the fluids in half, thus the value of masses of $m_{x}+m_{1 y}+m_{2 y}$ is divided by two $\left(\left(m_{x}+m_{1 y}+m_{2 y}\right) / 2\right)$ as the propellant enters each recycle valve. Resultantly, the density of the fluids flowing into both recycle valves is half the value of density $\rho_{s m}$ $\left(\rho_{s m} / 2\right)$. Therefore, density $\rho^{s}$ is half of density $\rho_{s m}\left(\rho^{s}=\left(\rho_{s m} / 2\right)\right)$ and is the density of the fluid egressing through each recycle valve. Density $\rho^{t}$ can be expressed as:

$$
\rho^{s}=\frac{\rho_{s m}}{2}=\frac{m_{x}+m_{1 y}+m_{2 y}}{2 v_{c}}
$$

The value of the density $\rho^{s}$ flowing into both recycle valves is given such that:

$$
\rho^{o}=3.53 \times 10^{-4} \mathrm{~kg} / \mathrm{m}^{\mathrm{a}}
$$

The propellant flowing into each recycle valves drives two turbines which generate a voltage $\varepsilon_{\text {recycle }}$. The equation of electromotor force $\varepsilon_{\text {recyele }}$ is given such that:

$$
\varepsilon_{\text {recycle }}=-\left(\frac{1}{y} \sqrt{\frac{2\left|F_{\text {sma }}\right|}{N_{B} A_{B} \rho^{f} \sin \theta_{B}}}\right) B A \sin \left(\left(\frac{1}{Y} \sqrt{\frac{2\left|F_{\text {gma }}\right|}{N_{B} A_{B} \rho^{f} \sin \theta_{B}}}\right) t\right)
$$

Applying the values of table 2 and associated quantities, the voltage generated by the turbines as the propellant egresses through the each recycle valve is given by:

$$
\varepsilon_{\text {recycle }}=-1.11 \text { Volts }
$$

Thus absolute value of voltage $\varepsilon_{\text {recycle }}$ is given such that:

$$
\left|\varepsilon_{\text {recyele }}\right|=1.11 \text { Volts }
$$

The corresponding value of potential energy denoted $2 e \varepsilon_{\text {recycle }}$ pertaining to both recycle valves takes on a value of:

$$
\left|2 e \varepsilon_{\text {recycle }}\right|=3.56 \times 10^{-19} \mathrm{~J}
$$

The total amount of electrical potential energy required to accelerate the ionized propellant out of all three nozzles is denoted $U_{\text {prop }}$ where $V_{\text {prop }}$ denotes the corresponding voltage value as expressed below

$$
U_{\text {prop }} \equiv e V_{\text {prop }}=V_{c} P_{\text {sm }}
$$


The combined dynamic pressure of all three fluid/ ionized propellant flows per time span $\Delta t$ suspended to the center of the enclosed chamber via electric force $F_{g W}$ generated by the chamber side walls is given by pressure $P_{S m}$. Due to the pressure energy relationship $\left(P=E / V_{c}\right)$, the energy required to generate a dynamic pressure value $P_{s m}$ is the product of pressure $P_{\mathscr{S m}}$ and the volume of the enclosed chamber $V_{c}\left(V_{c} P_{s m}\right)$ as shown in Eq.168. Thus, energy $U_{\text {prop }}$ can be expressed in terms of fluidic dynamic pressure such that:

$$
U_{\text {prop }}=\frac{V_{c} \rho_{x}\left(\mathbb{V}_{x x}\right)^{2}}{2}+V_{c}\left(\frac{\rho_{1 y}\left(\mathbb{V}_{1 y}\right)^{2}}{2}+\frac{\rho_{2 y}\left(\mathbb{V}_{2 y}\right)^{2}}{2}\right)
$$

Applying values from table 1 give a value of energy $U_{\text {prop }}$ such that:

$$
U_{\text {prop }}=2.5 \times 10^{-4} \mathrm{~J}
$$

In the task of calculating the management and movement of energy within the hypothetical OFFPC motor, the kinetic energy $k_{v}$ enacted on the entire vehicle is expressed by the equation below as derived in Eq.118 of section 6.

$$
k_{v}=\frac{(\Delta t)^{2}}{2\left(m_{v}+m_{x}+m_{1 y}+m_{2 y}\right)}\left[\left(A_{c}\left(\frac{\rho_{1 y}\left(V_{1 y}\right)^{2}}{2}+\frac{\rho_{2 y}\left(V_{2 y}\right)^{2}}{2}\right)\right)\right]^{2}
$$

Applying the associated values gives a value of vehicle kinetic energy $k_{w}$ such that:

$$
k_{\mathrm{v}}=7.66 \times 10^{-12} \mathrm{~J}
$$

As expressed in table 2, the voltage value $V_{\text {ema }}$ generated by the forward chamber wall to generate electric force $F_{\text {ema }}$ to temporarily trap the ionized propellant corresponds to an electric potential $e V_{e m a}$ which has a value of:

$$
e V_{e m a}=1.47 \times 10^{-5} \mathrm{~J}
$$

The sum of Electric potential energy values $\sum e V_{B}$ corresponding to voltage sum of $\sum V_{B}$ required for the generation of the repulsive (or positively charged) force $F_{\mathscr{G W}}$ of each side chamber of section 4 Eq.94 has a given value such that:

$$
\sum e V_{B}=2 \times 10^{-4} J
$$

Conclusively, the total electrical energy $E_{\text {output }}$ require to accelerate the ionized propellant out of all three nozzles, generate the repulsive force of the side chamber walls, and generate the adhesive force of the forward chamber wall is the sum of all three potential energy values $U_{p r o p}, e V_{e m \square}$, and $\sum e V_{B}$ as expressed below.

$$
E_{\text {output }}=U_{\text {prop }}+e V_{\text {ema }}+\sum e V_{B}
$$

Hence, applying the values of energies $U_{\text {prop }}, e V_{e m a}$, and $e V_{B}$ to Eq. 174 above gives a value of energy $E_{\text {output }}$ such that:

$$
E_{\text {output }}=4.65 \times 10^{-4} \mathrm{~J}
$$

The portion of energy recycled and converted to the kinetic energy of the entire vehicle is the sum of vehicle kinetic energy $k_{v}$ and the energy generated by the turbines within both recycle valves $\left|2 e \varepsilon_{\text {recycle }}\right|$ (i.e. $k_{v}+\left|2 e \varepsilon_{\text {recycle }}\right|$ ). This sum has a value such that:

$$
\left[k_{v}+\left|2 e \varepsilon_{\text {recycle }}\right|\right]=7.66 \times 10^{-12} J
$$

The portion of the energy that was not recycled or converted to vehicle kinetic energy ( $E_{\text {excess }}$ ) is the difference of energy output $E_{\text {output }}$ and energy sum $k_{v}+\left|2 e \varepsilon_{\text {recyele }}\right|$ as shown below. 


$$
E_{\text {excess }}=E_{\text {output }}-\left[k_{v}+\left|2 e \varepsilon_{\text {recycle }}\right|\right]
$$

Hence, the value of energy $E_{\text {excess }}$ is then given by:

$$
E_{\text {excess }} \approx 4.65 \times 10^{-4} \mathrm{~J}
$$

As expressed in Section 6, the energy that is not recycled or converted to vehicle kinetic energy is converted to thermal energy that is passed on from the propellant to the structure of the enclosed chamber and vehicle as the propellant contacts the forward chamber wall. In expressing the thermal energy that will be transferred from the propellant to the chamber structure, the average kinetic energy of the molecules of the ionized propellant is denoted $k_{t y}$ as expressed in Eq.123 of section 6. Molecular kinetic energy is expressed such that:

$$
k_{t y}=\frac{N m_{r}\left(v_{r m s}\right)^{2}}{2}=E_{\text {excess }}
$$

Thus we see that, molecular kinetic energy $k_{\text {ty }}$ is equal to energy $E_{\text {excess }}$ as expressed in Eq. 179 above, where $N$ is the number of molecules within the ionized propellant. Molecular number $N$ can be expressed such that:

$$
N=\left(\frac{m_{\text {tot }}}{M_{\text {mol }}}\right) \times N_{a}
$$

The propellant is composed of xenon gas which has a molecular weight of $132.906 \mathrm{~g} / \mathrm{mol}$ (i.e. $M_{\text {mol }}=132.906 \mathrm{~g} / \mathrm{mol}$ ) (Young \& Freedman,2004). The total mass $m_{\text {tot }}$ is the sum of masses $m_{x}, m_{1 y}$, and $m_{2 y}$ and has value of:

$$
m_{\text {tot }}=m_{x}+m_{1 y}+m_{2 y}=5 \times 10^{-15} \mathrm{~kg}
$$

The number of molecules composing the ionized propellant is given as:

$$
N \approx 2.26 \times 10^{7}
$$

The temperature increase imposed on the structure of the enclosed chamber is expressed in terms of molecular kinetic energy $k_{t y}$ such that:

$$
T=\frac{21}{a k}\left[k_{t y}\right] \equiv \frac{2}{a R n}\left[\frac{\mathrm{Nm}_{r}\left(\mathrm{w}_{\mathrm{rm}}\right)^{2}}{2}\right]
$$

Temperature $T$ can alternatively be expressed in terms of excess energy $E_{\text {excess }}$ such that:

$$
T=\frac{2}{a k}\left[E_{\text {EXcess }}\right]
$$

Applying the associated values of table 2 and the value of energy $E_{\text {excess }}$ gives a value of temperature $T$ such that:

$$
T=4.28 \times 10^{-27} K
$$

Thus, the value of Heat pertaining to the ionized propellant in terms of temperature $T$ is given such that:

$$
H_{\text {propellant }}=A_{p} e \sigma T^{4}
$$

Due to the performance figures used in this hypothetical demonstration, the value of $H_{\text {propellant }}$ is negligible, 
therefore, its value is excluded from this demonstration.

In conclusion, this mathematical demonstration of a hypothetical motor incorporating the theoretical OFFPC shows exorbitantly high voltage values for a very low thrust value as compared to the EM drive as well as other ion propulsion systems. However, it does show thrust values that are comparable to conventional ion propulsion systems with the advantage of a recyclable propellant. Moreover, the OFFPC is completely electrically dependent as with the EM drive due to the fact that the system does not lose mass as with conventional ion propulsion systems. The introduction of the OFFPC will hopefully inspire further research that will bring great improvements to its efficiency and to long duration space propulsion.

\section{Acknowledgements}

I would like to thank my Lord and Savior Jesus Christ for this achievement. Additionally, I would like to thank my father Edward Walker, my mother Juanita Walker-Kirkland, my sister Brittani Shingles, my brother Kenneth Walker, my nephew Robert Shingles, my cousin Adam Strickland, Mr. Eugene Thompson, and Mr. Ricky Stuart for their support.

\section{References}

Benson, T. (Editor), (2013). General Thrust equation, Glenn Research center Cleveland, Ohio 44135, retrieved from: http://www.grc.nasa.gov/WWW/k-12/airplane/thrsteq.html

Hyper Physics, (2017). Pressure, Georgia State University, retrieved from: http://hyperphysics.phy-astr.gsu.edu/hbase/press.html

Hyper Physics, (2017). Stefan-Boltzmann Law, Georgia State University, retrieved from: http://hyperphysics.phy-astr.gsu.edu/hbase/thermo/stefan.html

Nadis, S., (2014). Rocket man, Discover Magazine, Kalmbach Publishing Co., Waukesha, WI

NASA-Ion propulsion, (2016). Glenn Research Center Cleveland, Ohio 44135: Retrieved from: https://www.nasa.gov/centers/glenn/about/fs21grc.html

Penn state University (2017). Conservation of momentum using control volumes; lecture notes, Retrieved from: http://www.mne.psu.edu/cimbala/learning/fluid/cv_momentum/home.htm

Shawyer, R. (2006). A theory of microwave propulsion for spacecraft, SPR LTD., United Kingdom, Retrieved from: http://www.emdrive.com/theorypaper9-4.pdf

The physics classroom (2017). The logic behind momentum conservation, retrieved from http://www.physicsclassroom.com/class/momentum/u4l2b.cfm

Thompson, A., (2017). The science behind the impossible EM Drive, popular Mechanics, Retrieved from: http://www.popularmechanics.com/science/a24745/science-behind-em-drive/

Young, H., \& Freedman, A. R. (2004). Sears and Zemansky's University Physics, $11^{\text {th }}$ Edition, San Francisco, Cal., Pearson, Addison Wesley Pages 214, 227, 444-449, 516-518, 528-531, 806-07, 893, 908-913, 1023-1024, $1121-1124$

\section{Copyrights}

Copyright for this article is retained by the author(s), with first publication rights granted to the journal.

This is an open-access article distributed under the terms and conditions of the Creative Commons Attribution license which permits unrestricted use, distribution, and reproduction in any medium, provided the original work is properly cited. 